\title{
Coideal subalgebras in quantum affine algebras
}

\author{
A. I. Molev, E. Ragoucy and P. Sorba
}

\begin{abstract}
We introduce two subalgebras in the type $A$ quantum affine algebra which are coideals with respect to the Hopf algebra structure. In the classical limit $q \rightarrow 1$ each subalgebra specializes to the enveloping algebra $\mathrm{U}(\mathfrak{k})$, where $\mathfrak{k}$ is a fixed point subalgebra of the loop algebra $\mathfrak{g l}_{N}\left[\lambda, \lambda^{-1}\right]$ with respect to a natural involution corresponding to the embedding of the orthogonal or symplectic Lie algebra into $\mathfrak{g l}_{N}$. We also give an equivalent presentation of these coideal subalgebras in terms of generators and defining relations which have the form of reflection-type equations. We provide evaluation homomorphisms from these algebras to the twisted quantized enveloping algebras introduced earlier by Gavrilik and Klimyk and by Noumi. We also construct an analog of the quantum determinant for each of the algebras and show that its coefficients belong to the center of the algebra. Their images under the evaluation homomorphism provide a family of central elements of the corresponding twisted quantized enveloping algebra.
\end{abstract}

Preprint LAPTH-927/02

School of Mathematics and Statistics

University of Sydney, NSW 2006, Australia

alexm@maths.usyd.edu.au

LAPTH, Chemin de Bellevue, BP 110

F-74941 Annecy-le-Vieux cedex, France

ragoucy@lapp.in2p3.fr

LAPTH, Chemin de Bellevue, BP 110

F-74941 Annecy-le-Vieux cedex, France

sorba@lapp.in2p3.fr 


\section{Introduction}

For a simple Lie algebra $\mathfrak{g}$ over $\mathbb{C}$ consider the corresponding quantized enveloping algebra $\mathrm{U}_{q}(\mathfrak{g})$; see Drinfeld [9], Jimbo [18. If $\mathfrak{k}$ is a subalgebra of $\mathfrak{g}$ then $\mathrm{U}(\mathfrak{k})$ is a Hopf subalgebra of $\mathrm{U}(\mathfrak{g})$. However, $\mathrm{U}_{q}(\mathfrak{k})$, even when it is defined, need not be isomorphic to a Hopf subalgebra of $\mathrm{U}_{q}(\mathfrak{g})$. In the case where $(\mathfrak{g}, \mathfrak{k})$ is a classical symmetric pair the twisted quantized enveloping algebra $\mathrm{U}_{q}^{\mathrm{tw}}(\mathfrak{k})$ was introduced by Noumi [31] (type $A$ pairs) and by Noumi and Sugitani 32] (remaining classical types). This is a subalgebra and a left coideal of the Hopf algebra $\mathrm{U}_{q}(\mathfrak{g})$ which specializes to $\mathrm{U}(\mathfrak{k})$ as $q \rightarrow 1$. The algebras $\mathrm{U}_{q}^{\text {tw }}(\mathfrak{k})$ play an important role in the theory of quantum symmetric spaces developed in [31] and [32]. In particular, in the type $A$, which we are only concerned with in this paper, there are two twisted quantized enveloping algebras $\mathrm{U}_{q}^{\mathrm{tw}}\left(\mathfrak{o}_{N}\right)$ and $\mathrm{U}_{q}^{\mathrm{tw}}\left(\mathfrak{s p}_{2 n}\right)$ corresponding to the symmetric pairs

$$
\begin{array}{cl}
\text { AI : } & \left(\mathfrak{g l}_{N}, \mathfrak{o}_{N}\right), \\
\text { AII : } & \left(\mathfrak{g l}_{2 n}, \mathfrak{s p}_{2 n}\right),
\end{array}
$$

respectively. It was also shown by Noumi [3] that the algebra $\mathrm{U}_{q}^{\mathrm{tw}}\left(\mathfrak{o}_{N}\right)$ coincides with the one introduced earlier by Gavrilik and Klimyk [12]. The algebra $\mathrm{U}_{q}^{\mathrm{tw}}\left(\mathfrak{o}_{N}\right)$ also appears as the symmetry algebra for the $q$-oscillator representation of the quantized enveloping algebra $\mathrm{U}_{q}\left(\mathfrak{s p}_{2 n}\right)$; see Noumi, Umeda and Wakayama [33, 34].

In Noumi's approach, the defining relations for the quantized algebras can be written in the form of a reflection-type equation. A constant solution of the reflection equation provides an embedding of the twisted quantized enveloping algebra into $\mathrm{U}_{q}\left(\mathfrak{g l}_{N}\right)$. The quantum homogeneous spaces corresponding to the remaining series of the classical symmetric pairs of type $A$

$$
\text { AIII : } \quad\left(\mathfrak{g l}_{N}, \mathfrak{g l}_{N-l} \oplus \mathfrak{g l}_{l}\right)
$$

were studied by Dijkhuizen, Noumi and Sugitani [6], [7]. A one-parameter family of the constant solutions of the appropriate reflection equation was produced in [7], although no reflection-type presentation of the subalgebras of type $\mathrm{U}_{q}^{\text {tw }}(\mathfrak{k})$ were formally introduced.

A different description of the coideal subalgebras of $\mathrm{U}_{q}(\mathfrak{g})$ associated with an arbitrary irreducible symmetric pair $(\mathfrak{g}, \mathfrak{k})$ was given by Letzter [22, 23]. The subalgebras are presented by generators and explicit relations depending on the Cartan matrix of $\mathfrak{g}$; see [23]. In particular, this work demonstrates the importance of the coideal property: it makes the construction of the twisted quantized algebras essentially unique.

Natural infinite-dimensional analogs of the symmetric pairs are provided by involutive subalgebras in the polynomial current Lie algebras $\mathfrak{g}[x]=\mathfrak{g} \otimes \mathbb{C}[x]$ or loop 
algebras $\mathfrak{g}\left[\lambda, \lambda^{-1}\right]=\mathfrak{g} \otimes \mathbb{C}\left[\lambda, \lambda^{-1}\right]$. Let $(\mathfrak{g}, \mathfrak{k})$ be a symmetric pair and $\mathfrak{g}=\mathfrak{k} \oplus \mathfrak{p}$ be the decomposition determined by the involution $\theta$ of $\mathfrak{g}$. So, $\mathfrak{k}$ and $\mathfrak{p}$ are the eigenspaces of $\theta$ with the eigenvalues 1 and -1 , respectively. Then the twisted polynomial current Lie algebra $\mathfrak{g}[x]^{\theta}$ can be defined by

$$
\mathfrak{g}[x]^{\theta}=\mathfrak{k} \oplus \mathfrak{p} x \oplus \mathfrak{k} x^{2} \oplus \mathfrak{p} x^{3} \oplus \cdots,
$$

or, equivalently, it is the fixed point subalgebra of $\mathfrak{g}[x]$ with respect to the extension of $\theta$ given by

$$
\theta: A x^{p} \mapsto(-1)^{p} \theta(A) x^{p}, \quad A \in \mathfrak{g} .
$$

As it was demonstrated by Drinfeld [9], the enveloping algebra $\mathrm{U}(\mathfrak{g}[x])$ admits a canonical deformation in the class of Hopf algebras. The corresponding "quantum" algebra is called the Yangian and denoted by $\mathrm{Y}(\mathfrak{g})$. For the case where $\theta$ is an involution of $\mathfrak{g l}_{N}$ corresponding to the pair of type AI or AII, quantum analogs of the symmetric pairs $\left(\mathfrak{g l}_{N}[x], \mathfrak{g l}_{N}[x]^{\theta}\right)$ are provided by the Olshanski twisted Yangians 35]. These are coideal subalgebras in the Yangian $\mathrm{Y}\left(\mathfrak{g l}_{N}\right)$ and each of them is a deformation of the enveloping algebra $\mathrm{U}\left(\mathfrak{g l}_{N}[x]^{\theta}\right)$.

In the AIII case, the quantum analogs of the pairs $\left(\mathfrak{g l}_{N}[x], \mathfrak{g l}_{N}[x]^{\theta}\right)$ are provided by the reflection algebras $B(N, l)$ which are coideal subalgebras of $\mathrm{Y}\left(\mathfrak{g l}_{N}\right)$ originally introduced by Sklyanin [37]. Recently, these algebras and their representations were studied in connection with the NLS model; see Liguori, Mintchev and Zhao 24, Mintchev, Ragoucy and Sorba [25], Molev and Ragoucy [29].

For the symmetric pairs $\left(\mathfrak{g}[x], \mathfrak{g}[x]^{\theta}\right)$ of general types the corresponding coideal subalgebras in the Yangian $\mathrm{Y}(\mathfrak{g})$ were recently introduced by Delius, MacKay and Short 4] in relation with the principal chiral models with boundaries. These subalgebras are given in terms of the $Q$-presentation of the Yangian. A different $R$-matrix presentation of coideal subalgebras in the (super) Yangian $\mathrm{Y}(\mathfrak{g})$ is given by Arnaudon, Avan, Crampé, Frappat and Ragoucy [1]. Field theoretical applications of the coideal subalgebras in the quantum affine algebras have been studied in a recent paper by Delius and MacKay [5].

In the case of the loop algebra $\widehat{\mathfrak{g}}=\mathfrak{g}\left[\lambda, \lambda^{-1}\right]$ there is another natural way (cf. (1.4) ) to extend the involution $\theta$ of $\mathfrak{g}$,

$$
\theta: A \lambda^{p} \mapsto \theta(A) \lambda^{-p}, \quad A \in \mathfrak{g}
$$

and thus to define the fixed point subalgebra $\widehat{\mathfrak{g}}^{\theta}$. In this paper we introduce certain quantizations of the symmetric pairs $\left(\widehat{\mathfrak{g l}}_{N}, \widehat{\mathfrak{g l}}_{N}^{\theta}\right)$ associated with the involution $\theta$ corresponding to the pairs AI and AII. We define the twisted $q$-Yangians $\mathrm{Y}_{q}^{\mathrm{tw}}\left(\mathfrak{o}_{N}\right)$ and $\mathrm{Y}_{q}^{\mathrm{tw}}\left(\mathfrak{s p}_{2 n}\right)$ as subalgebras of the quantum affine algebra $\mathrm{U}_{q}\left(\widehat{\mathfrak{g l}}_{N}\right)$. They are left coideals with respect to the coproduct on $\mathrm{U}_{q}\left(\widehat{\mathfrak{g l}}_{N}\right)$ and specialize to $\mathrm{U}\left(\widehat{\mathfrak{g l}}_{N}^{\theta}\right)$ as $q \rightarrow 1$. 
At this point we consider it necessary to comment on the terminology. Although, as we have mentioned above, the Lie algebra $\widehat{\mathfrak{g l}}_{N}^{\theta}$ is not a "twisted" quantum affine algebra in the usual meaning, we believe the names we use for the coideal subalgebras can be justified having in mind their analogy with both the twisted Yangians and the $q$-Yangian; cf. [30]. The latter is a subalgebra of the quantum affine algebra $\mathrm{U}_{q}\left(\widehat{\mathfrak{g l}}_{N}\right)$ which can be regarded as a $q$-analog of the usual Yangian $\mathrm{Y}\left(\mathfrak{g l}_{N}\right)$; see also Section 3 below.

Our first main result is a construction of the evaluation homomorphisms

$$
\mathrm{Y}_{q}^{\mathrm{tw}}\left(\mathfrak{o}_{N}\right) \rightarrow \mathrm{U}_{q}^{\mathrm{tw}}\left(\mathfrak{o}_{N}\right), \quad \mathrm{Y}_{q}^{\mathrm{tw}}\left(\mathfrak{s p}_{2 n}\right) \rightarrow \mathrm{U}_{q}^{\mathrm{tw}}\left(\mathfrak{s p}_{2 n}\right)
$$

to the corresponding twisted quantized enveloping algebras of [12] and [31]. Note that an evaluation homomorphism $\mathrm{U}_{q}(\widehat{\mathfrak{g}}) \rightarrow \mathrm{U}_{q}(\mathfrak{g})$ from the quantum affine algebra to the corresponding quantized enveloping algebra only exists if $\mathfrak{g}$ is of $A$ type, and the same holds for the case of the Yangians; see Jimbo [19], Drinfeld [9]. In both the cases the evaluation homomorphisms play an important role in the representation theory of the quantum algebras; see Chari and Pressley [2]. An evaluation homomorphism from the twisted Yangian to the corresponding enveloping algebra $\mathrm{U}\left(\mathfrak{o}_{N}\right)$ or $\mathrm{U}\left(\mathfrak{s p}_{2 n}\right)$ does exist (see [35], 28]) and has many applications in the classical representation theory; see e.g. 27] for an overview. Note also that the existence of the homomorphisms (1.6) is not directly related with the corresponding fact for the $A$ type algebras but is quite a nontrivial property of the reflection equations satisfied by the generators of the twisted $q$-Yangians.

Next we construct an analog of the quantum determinant for each twisted $q$ Yangian and show that its coefficients belong to the center of this algebra. The application of the evaluation homomorphism (1.6) yields a family of central elements in $\mathrm{U}_{q}^{\mathrm{tw}}\left(\mathfrak{o}_{N}\right)$ and $\mathrm{U}_{q}^{\mathrm{tw}}\left(\mathfrak{s p}_{2 n}\right)$. In the orthogonal case we also produce a 'short' determinantlike formula for this analog which employs a certain map from the symmetric group into itself. This same map was used in the short formulas for the Sklyanin determinants for the twisted Yangians; see [27. Some other families of Casimir elements were constructed by Noumi, Umeda and Wakayama [34] and by Gavrilik and Iorgov [13. It would be interesting to understand the relationship between the families, as well as to investigate possible applications to the study of the quantum Howe dual pairs; cf. [33, 34.

Another intriguing problem is to construct coideal subalgebras of $\mathrm{U}_{q}\left(\widehat{\mathfrak{g l}}_{N}\right)$ of type AIII, i.e., to find $q$-analogs of the Sklyanin reflection algebras $B(N, l)$ mentioned above.

We are grateful to Gustav Delius, Masatoshi Noumi and Tôru Umeda for valuable discussions. The financial support of the Australian Research Council and the 
Laboratoire d'Annecy-le-Vieux de Physique Théorique is acknowledged.

\section{Coideal subalgebras of $\mathrm{U}_{q}\left(\mathfrak{g l}_{N}\right)$}

We shall use an $R$-matrix presentation of the algebra $\mathrm{U}_{q}\left(\mathfrak{g l}_{N}\right)$. Our main references are Jimbo [19] and Reshetikhin, Takhtajan and Faddeev [36. We fix a complex parameter $q$ which is nonzero and not a root of unity. Consider the $R$-matrix

$$
R=q \sum_{i} E_{i i} \otimes E_{i i}+\sum_{i \neq j} E_{i i} \otimes E_{j j}+\left(q-q^{-1}\right) \sum_{i<j} E_{i j} \otimes E_{j i}
$$

which is an element of End $\mathbb{C}^{N} \otimes$ End $\mathbb{C}^{N}$, where the $E_{i j}$ denote the standard matrix units and the indices run over the set $\{1, \ldots, N\}$. The $R$-matrix satisfies the YangBaxter equation

$$
R_{12} R_{13} R_{23}=R_{23} R_{13} R_{12},
$$

where both sides take values in End $\mathbb{C}^{N} \otimes$ End $\mathbb{C}^{N} \otimes$ End $\mathbb{C}^{N}$ and the subindices indicate the copies of End $\mathbb{C}^{N}$, e.g., $R_{12}=R \otimes 1$ etc.

The quantized enveloping algebra $\mathrm{U}_{q}\left(\mathfrak{g l}_{N}\right)$ is generated by elements $t_{i j}$ and $\bar{t}_{i j}$ with $1 \leq i, j \leq N$ subject to the relations ${ }^{1}$

$$
\begin{array}{rlrl}
t_{i j} & =\bar{t}_{j i}=0, & 1 \leq i<j \leq N, & \\
t_{i i} \bar{t}_{i i} & =\bar{t}_{i i} t_{i i}=1, & 1 \leq i \leq N, \\
R T_{1} T_{2} & =T_{2} T_{1} R, \quad R \bar{T}_{1} \bar{T}_{2}=\bar{T}_{2} \bar{T}_{1} R, \quad R \bar{T}_{1} T_{2}=T_{2} \bar{T}_{1} R .
\end{array}
$$

Here $T$ and $\bar{T}$ are the matrices

$$
T=\sum_{i, j} t_{i j} \otimes E_{i j}, \quad \bar{T}=\sum_{i, j} \bar{t}_{i j} \otimes E_{i j},
$$

which are regarded as elements of the algebra $\mathrm{U}_{q}\left(\mathfrak{g l}_{N}\right) \otimes$ End $\mathbb{C}^{N}$. Both sides of each of the $R$-matrix relations in (2.3) are elements of $\mathrm{U}_{q}\left(\mathfrak{g l}_{N}\right) \otimes$ End $\mathbb{C}^{N} \otimes$ End $\mathbb{C}^{N}$ and the subindices of $T$ and $\bar{T}$ indicate the copies of End $\mathbb{C}^{N}$ where $T$ or $\bar{T}$ acts; e.g. $T_{1}=T \otimes 1$. In terms of the generators the defining relations between the $t_{i j}$ can be written as

$$
q^{\delta_{i j}} t_{i a} t_{j b}-q^{\delta_{a b}} t_{j b} t_{i a}=\left(q-q^{-1}\right)\left(\delta_{b<a}-\delta_{i<j}\right) t_{j a} t_{i b},
$$

where $\delta_{i<j}$ equals 1 if $i<j$ and 0 otherwise. The relations between the $\bar{t}_{i j}$ are obtained by replacing $t_{i j}$ by $\bar{t}_{i j}$ everywhere in (2.5). Finally, the relations involving both $t_{i j}$ and $\bar{t}_{i j}$ have the form

$$
q^{\delta_{i j}} \bar{t}_{i a} t_{j b}-q^{\delta_{a b}} t_{j b} \bar{t}_{i a}=\left(q-q^{-1}\right)\left(\delta_{b<a} t_{j a} \bar{t}_{i b}-\delta_{i<j} \bar{t}_{j a} t_{i b}\right) .
$$

\footnotetext{
${ }^{1}$ Our $T$ and $\bar{T}$ correspond to the $L$-operators $L^{-}$and $L^{+}$, respectively, in the notation of [36].
} 
We shall also use another $R$-matrix $\widetilde{R}$ given by

$$
\widetilde{R}=q^{-1} \sum_{i} E_{i i} \otimes E_{i i}+\sum_{i \neq j} E_{i i} \otimes E_{j j}+\left(q^{-1}-q\right) \sum_{i>j} E_{i j} \otimes E_{j i} .
$$

We have the relations

$$
R-\widetilde{R}=\left(q-q^{-1}\right) P, \quad \widetilde{R}=P R^{-1} P,
$$

where

$$
P=\sum_{i, j} E_{i j} \otimes E_{j i}
$$

is the permutation operator. The following relations are implied by (2.3)

$$
\widetilde{R} T_{1} T_{2}=T_{2} T_{1} \widetilde{R}, \quad \widetilde{R} \bar{T}_{1} \bar{T}_{2}=\bar{T}_{2} \bar{T}_{1} \widetilde{R}, \quad \widetilde{R} T_{1} \bar{T}_{2}=\bar{T}_{2} T_{1} \widetilde{R} .
$$

The coproduct $\Delta$ on $\mathrm{U}_{q}\left(\mathfrak{g l}_{N}\right)$ is defined by the relations

$$
\Delta\left(t_{i j}\right)=\sum_{k=1}^{N} t_{i k} \otimes t_{k j}, \quad \Delta\left(\bar{t}_{i j}\right)=\sum_{k=1}^{N} \bar{t}_{i k} \otimes \bar{t}_{k j} .
$$

It is well known that the algebra $\mathrm{U}_{q}\left(\mathfrak{g l}_{N}\right)$ specializes to $\mathrm{U}\left(\mathfrak{g l}_{N}\right)$ as $q \rightarrow 1$. To make this more precise, regard $q$ as a formal variable and $\mathrm{U}_{q}\left(\mathfrak{g l}_{N}\right)$ as an algebra over $\mathbb{C}(q)$. Then set $\mathcal{A}=\mathbb{C}\left[q, q^{-1}\right]$ and consider the $\mathcal{A}$-subalgebra $\mathrm{U}_{\mathcal{A}}$ of $\mathrm{U}_{q}\left(\mathfrak{g l}_{N}\right)$ generated by the elements

$$
\tau_{i j}=\frac{t_{i j}}{q-q^{-1}} \quad \text { for } \quad i>j, \quad \bar{\tau}_{i j}=\frac{\bar{t}_{i j}}{q-q^{-1}} \quad \text { for } \quad i<j,
$$

and

$$
\tau_{i i}=\frac{t_{i i}-1}{q-1}, \quad \bar{\tau}_{i i}=\frac{\bar{t}_{i i}-1}{q-1},
$$

for $i=1, \ldots, N$. Then we have an isomorphism

$$
\mathrm{U}_{\mathcal{A}} \otimes_{\mathcal{A}} \mathbb{C} \cong \mathrm{U}\left(\mathfrak{g l}_{N}\right)
$$

with the action of $\mathcal{A}$ on $\mathbb{C}$ defined via the evaluation $q=1$; see e.g. [2, Section 9.2]. Note that $\tau_{i j}$ and $\bar{\tau}_{i j}$ respectively specialize to the elements $E_{i j}$ and $-E_{i j}$ of $\mathrm{U}\left(\mathfrak{g l}_{N}\right)$. More generally, given a subalgebra $V$ of $\mathrm{U}_{q}\left(\mathfrak{g l}_{N}\right)$, set $V_{\mathcal{A}}=V \cap \mathrm{U}_{\mathcal{A}}$. Following Letzter [22, Section 1], we shall say that $V$ specializes to the subalgebra $V^{\circ}$ of $\mathrm{U}\left(\mathfrak{g l}_{N}\right)$ (as $q$ goes to 1 ) if the image of $V_{\mathcal{A}}$ in $\mathrm{U}_{\mathcal{A}} \otimes_{\mathcal{A}} \mathbb{C}$ is $V^{\circ}$. 


\subsection{Orthogonal case}

Following Noumi [31], we introduce the twisted quantized enveloping algebra $\mathrm{U}_{q}^{\mathrm{tw}}\left(\mathfrak{o}_{N}\right)$ as the subalgebra of $\mathrm{U}_{q}\left(\mathfrak{g l}_{N}\right)$ generated by the matrix elements of the matrix $S=$ $T \bar{T}^{t}$. It can be easily derived from (2.3) (see [31]) that the matrix $S$ satisfies the relations

$$
\begin{gathered}
s_{i j}=0, \quad 1 \leq i<j \leq N, \\
s_{i i}=1, \quad 1 \leq i \leq N, \\
R S_{1} R^{t} S_{2}=S_{2} R^{t} S_{1} R,
\end{gathered}
$$

where $R^{t}:=R^{t_{1}}$ denotes the element obtained from $R$ by the transposition in the first tensor factor:

$$
R^{t}=q \sum_{i} E_{i i} \otimes E_{i i}+\sum_{i \neq j} E_{i i} \otimes E_{j j}+\left(q-q^{-1}\right) \sum_{i<j} E_{j i} \otimes E_{j i}
$$

Indeed, the only nontrivial part of this derivation is to verify that

$$
R T_{1} \bar{T}_{1}^{t} R^{t} T_{2} \bar{T}_{2}^{t}=T_{2} \bar{T}_{2}^{t} R^{t} T_{1} \bar{T}_{1}^{t} R
$$

However, this is implied by the relation $R R^{t}=R^{t} R$ and the following consequences of (2.3):

$$
\bar{T}_{1}^{t} R^{t} T_{2}=T_{2} R^{t} \bar{T}_{1}^{t}, \quad R \bar{T}_{1}^{t} \bar{T}_{2}^{t}=\bar{T}_{2}^{t} \bar{T}_{1}^{t} R .
$$

We now prove an auxiliary lemma which establishes a weak form of the PoincaréBirkhoff-Witt theorem for abstract algebras defined by the relation (2.17). It will be used in both the orthogonal and symplectic cases.

Lemma 2.1. Consider the associative algebra with $N^{2}$ generators $s_{i j}, i, j=1, \ldots, N$ and the defining relations written in terms of the matrix $S=\left(s_{i j}\right)$ by the relation (2.17). Then the ordered monomials of the form

$$
s_{11}^{k_{11}} s_{12}^{k_{12}} \cdots s_{1 N}^{k_{1 N}} \cdots s_{N 1}^{k_{N 1}} s_{N 2}^{k_{N 2}} \cdots s_{N N}^{k_{N N}}
$$

with nonnegative powers $k_{i j}$ linearly span the algebra.

Proof. Rewriting (2.17) in terms of the generators we get

$$
\begin{aligned}
q^{\delta_{a j}+\delta_{i j}} s_{i a} s_{j b}-q^{\delta_{a b}+\delta_{i b}} s_{j b} s_{i a} & =\left(q-q^{-1}\right) q^{\delta_{a i}}\left(\delta_{b<a}-\delta_{i<j}\right) s_{j a} s_{i b} \\
& +\left(q-q^{-1}\right)\left(q^{\delta_{a b}} \delta_{b<i} s_{j i} s_{b a}-q^{\delta_{i j}} \delta_{a<j} s_{i j} s_{a b}\right) \\
& +\left(q-q^{-1}\right)^{2}\left(\delta_{b<a<i}-\delta_{a<i<j}\right) s_{j i} s_{a b},
\end{aligned}
$$


where $\delta_{i<j}$ or $\delta_{i<j<k}$ equals 1 if the subindex inequality is satisfied and 0 otherwise. We shall be proving that any monomial in the generators can be written as a linear combination of monomials of the form (2.21). Given a monomial

$$
s_{i_{1} a_{1}} \cdots s_{i_{p} a_{p}}
$$

we introduce its length $p$ and weight $w$ by $w=i_{1}+\cdots+i_{p}$. Clearly, for a monomial of length $p$ the weight can range between $p$ and $p N$. We shall use induction on $w$. By (2.22) we obtain the following equality modulo products of weight less than $i+j$ : for $i \geq j$

$$
q^{\delta_{a j}+\delta_{i j}} s_{i a} s_{j b} \equiv q^{\delta_{a b}+\delta_{i b}} s_{j b} s_{i a}+\delta_{b<a}\left(q-q^{-1}\right) q^{\delta_{a i}} s_{j a} s_{i b} .
$$

This allows us to represent (2.23) modulo monomials of weight less than $w$ as a linear combination of monomials $s_{j_{1} b_{1}} \cdots s_{j_{p} b_{p}}$ of weight $w$ such that $j_{1} \leq \cdots \leq j_{p}$. Consider a submonomial $s_{i c_{1}} \cdots s_{i c_{r}}$ containing generators with the same first index. By (2.24) we have for $a>b$

$$
q^{\delta_{a i}+1} s_{i a} s_{i b} \equiv q^{\delta_{a b}+\delta_{i b}} s_{i b} s_{i a} .
$$

Using this relation repeatedly we bring the submonomial to the required form.

We shall be proving now that (2.15) -(2.17) are precisely the defining relations of the algebra $\mathrm{U}_{q}^{\text {tw }}\left(\mathfrak{o}_{N}\right)$. In other words, the following theorem takes place.

Theorem 2.2. The abstract algebra $\mathcal{S}$ generated by elements $s_{i j}, i, j=1, \ldots, N$ with the defining relations (2.15) -(2.17) is isomorphic to $\mathrm{U}_{q}^{\mathrm{tw}}\left(\mathfrak{o}_{N}\right)$.

Proof. It was mentioned in [34, Remark 7.9(1)] without a detailed proof that this fact can be established with the use of the Diamond Lemma. We employ a different approach based on a weak Poincaré-Birkhoff-Witt theorem for the algebra $\mathcal{S}$.

For this proof only, denote the matrix $T \bar{T}^{t}$ by $\widetilde{S}$ and its matrix elements by $\widetilde{s}_{i j}$. As we noted above, the map $S \mapsto \widetilde{S}$ defines an algebra homomorphism $\varphi: \mathcal{S} \rightarrow \mathrm{U}_{q}^{\text {tw }}\left(\mathfrak{o}_{N}\right)$. We only need to show that this homomorphism is injective. By Lemma 2.1] the monomials

$$
s_{21}^{k_{21}} s_{31}^{k_{31}} s_{32}^{k_{32}} \cdots s_{N 1}^{k_{N 1}} s_{N 2}^{k_{N 2}} \cdots s_{N, N-1}^{k_{N, N-1}}
$$

span the algebra $\mathcal{S}$. We shall show that the images of these monomials under $\varphi$ are linearly independent. We prove, in fact, that given any linear ordering on the set of generators $\widetilde{s}_{i j}, i>j$, the ordered monomials in the $\widetilde{s}_{i j}$ are linearly independent. Regarding $q$ as a formal variable, we keep the notation $\mathcal{A}$ for the algebra of Laurent polynomials $\mathbb{C}\left[q, q^{-1}\right]$; see Section 2, Set $V=\mathrm{U}_{q}^{\text {tw }}\left(\mathfrak{o}_{N}\right)$ and note that the subalgebra $V_{\mathcal{A}}$ is generated by the elements $\sigma_{i j}:=\widetilde{s}_{i j} /\left(q-q^{-1}\right)$ with $i>j$. It is enough to verify 
that the ordered monomials in the generators $\sigma_{i j}$ are linearly independent over $\mathcal{A}$. We have for $i>j$

$$
\sigma_{i j}=\tau_{i j}+\bar{\tau}_{j i}+(q-1)\left(\tau_{i i} \bar{\tau}_{j i}+\tau_{i j} \bar{\tau}_{j j}\right)+\left(q-q^{-1}\right) \sum_{j<a<i} \tau_{i a} \bar{\tau}_{j a} .
$$

Therefore, the image of $\sigma_{i j}$ in $V_{\mathcal{A}} \otimes_{\mathcal{A}} \mathbb{C}$ is $F_{i j}:=E_{i j}-E_{j i}$. The elements $F_{i j}$ with $i>j$ constitute a basis of a subalgebra of $\mathfrak{g l}_{N}$ isomorphic to the orthogonal Lie algebra $\mathfrak{o}_{N}$. By the Poincaré-Birkhoff-Witt theorem for $\mathfrak{o}_{N}$ the ordered monomials in the $F_{i j}$ are linearly independent. Now suppose, on the contrary, that there exists a nontrivial linear combination of the ordered monomials in the $\sigma_{i j}$ equal to zero:

$$
\sum_{(k)} c_{(k)} \prod_{i>j} \sigma_{i j}^{k_{i j}}=0,
$$

summed over the multi-indices $(k)=\left(k_{i j} \mid i>j\right)$, where $c_{(k)} \in \mathcal{A}$. We may assume that at least one coefficient $c_{(k)}$ in (2.28) does not vanish at $q=1$. Taking the image of (2.28) in $V_{\mathcal{A}} \otimes_{\mathcal{A}} \mathbb{C}$ yields a nontrivial linear combination of the ordered monomials in the $F_{i j}$ equal to zero. This makes a contradiction proving the claim.

As a corollary of the above argument we obtain an analog of the PoincaréBirkhoff-Witt theorem for the algebra $\mathcal{S}$. A different proof was given by N. Iorgov; see [15].

Corollary 2.3. The monomials (2.26) constitute a basis of the algebra $\mathcal{S}$.

It follows from the proof of the theorem that the algebra $V_{\mathcal{A}} \otimes_{\mathcal{A}} \mathbb{C}$ coincides with the enveloping algebra $\mathrm{U}\left(\mathfrak{o}_{N}\right)$. Thus, $\mathrm{U}_{q}^{\text {tw }}\left(\mathfrak{o}_{N}\right)$ specializes to $\mathrm{U}\left(\mathfrak{o}_{N}\right)$ as $q \rightarrow 1$; see [12] and [31, Section 2.4]. This result also follows from [21, Section 6].

Regarding $\mathrm{U}_{q}^{\mathrm{tw}}\left(\mathfrak{o}_{N}\right)$ as a subalgebra of $\mathrm{U}_{q}\left(\mathfrak{g l}_{N}\right)$ we introduce another matrix $\bar{S}$ by

$$
\bar{S}=\bar{T} T^{t}
$$

The matrix $\bar{S}=\left(\bar{s}_{i j}\right)$ is upper triangular with 1's on the diagonal. It is related to $S$ by the formula

$$
\bar{S}=1-q+q S^{t}
$$

or, in terms of the matrix elements, $\bar{s}_{i j}=q s_{j i}$ for $i<j$. Indeed, (2.3) implies

$$
\bar{t}_{i a} t_{j a}=q t_{j a} \bar{t}_{i a} .
$$

Taking the sum over $a$ gives the result. So, the elements $\bar{s}_{i j}$ belong to the subalgebra $\mathrm{U}_{q}^{\mathrm{tw}}\left(\mathfrak{o}_{N}\right)$

The next proposition is immediate from (2.11). It shows that the subalgebra $\mathrm{U}_{q}^{\mathrm{tw}}\left(\mathfrak{o}_{N}\right)$ is a left coideal of $\mathrm{U}_{q}\left(\mathfrak{g l}_{N}\right)$. This property was mentioned in [31, Section 2.4] and proofs can be found in [14] and [21, Lemma 6.3]. 
Proposition 2.4. The image of the generator $s_{i j}$ of $\mathrm{U}_{q}^{\mathrm{tw}}\left(\mathfrak{o}_{N}\right)$ under the coproduct is given by

$$
\Delta\left(s_{i j}\right)=\sum_{k, l=1}^{N} t_{i k} \bar{t}_{j l} \otimes s_{k l} .
$$

Remark 2.5. It was shown in 31] that for any nondegenerate diagonal matrix $D$ the matrix $S=T D \bar{T}^{t}$ satisfies the reflection equation (2.17). Therefore one can define a family of subalgebras in $\mathrm{U}_{q}\left(\mathfrak{g l}_{N}\right)$ parametrized by the matrices $D$. However, all of them are isomorphic to each other as abstract algebras which can be seen from the fact that for any diagonal matrix $C$ the relation (2.17) is preserved by the transformation $S \mapsto C S C$. Indeed, the entries of $S$ are then transformed as $s_{i j} \mapsto s_{i j} c_{i} c_{j}$ and the claim is immediate from (2.22). The corresponding remark also applies to the symplectic case considered below.

\subsection{Symplectic case}

Following again [31] introduce the $2 n \times 2 n$ matrix $G$ by

$$
G=q \sum_{k=1}^{n} E_{2 k-1,2 k}-\sum_{k=1}^{n} E_{2 k, 2 k-1} .
$$

It is convenient to introduce the involution of the set of indices $\{1, \ldots, 2 n\}$ which we denote by a prime and which acts by the rule: for $k=1, \ldots, n$

$$
(2 k-1)^{\prime}=2 k \quad \text { and } \quad(2 k)^{\prime}=2 k-1 .
$$

To introduce the twisted quantized enveloping algebra $\mathrm{U}_{q}^{\mathrm{tw}}\left(\mathfrak{s p}_{2 n}\right)$ consider the matrix $S=T G \bar{T}^{t}$ with entries in $\mathrm{U}_{q}\left(\mathfrak{g l}_{2 n}\right)$. For each odd $i=1,3, \ldots, 2 n-1$ the element $s_{i i^{\prime}}=q t_{i i} \bar{t}_{i^{\prime} i^{\prime}}$ is invertible in $\mathrm{U}_{q}\left(\mathfrak{g l}_{2 n}\right)$. We define $\mathrm{U}_{q}^{\mathrm{tw}}\left(\mathfrak{s p}_{2 n}\right)$ as the subalgebra of $\mathrm{U}_{q}\left(\mathfrak{g l}_{2 n}\right)$ generated by the matrix elements of the matrix $S$ and by the elements ${ }^{2} s_{i i^{\prime}}^{-1}$, $i=1,3, \ldots, 2 n-1$. Clearly, the matrix $S$ has a block-triangular form with $n$ diagonal $2 \times 2$-blocks so that

$$
s_{i j}=0 \quad \text { for } \quad i<j \quad \text { with } \quad j \neq i^{\prime}
$$

As in the orthogonal case, the matrix $S$ satisfies the relation

$$
R S_{1} R^{t} S_{2}=S_{2} R^{t} S_{1} R
$$

\footnotetext{
${ }^{2}$ These additional generators, not considered in [31, will bring some simplification in the arguments below.
} 
with the same definition of $R$ and $R^{t}$ (taking $N=2 n$ ), cf. (2.15)-(2.17). This follows from the fact that $G$ is a solution of the reflection equation 31 .

$$
R G_{1} R^{t} G_{2}=G_{2} R^{t} G_{1} R
$$

Lemma 2.6. For any odd $i=1,3, \ldots, 2 n-1$ we have the identity

$$
s_{i^{\prime} i^{\prime}} s_{i i}-q^{2} s_{i^{\prime} i} s_{i i^{\prime}}=q^{3} .
$$

Proof. The calculation is the same for each $i$ so we take $i=1$. We have

$$
\begin{array}{ll}
s_{11}=q t_{11} \bar{t}_{12}, & s_{12}=q t_{11} \bar{t}_{22} \\
s_{22}=q t_{21} \bar{t}_{22}, & s_{21}=q t_{21} \bar{t}_{12}-t_{22} \bar{t}_{11} .
\end{array}
$$

Now the relation is implied by the defining relations (2.3) in $\mathrm{U}_{q}\left(\mathfrak{g l}_{N}\right)$.

Next we prove an analog of Theorem 2.2 for the algebra $\mathrm{U}_{q}^{\mathrm{tw}}\left(\mathfrak{s p}_{2 n}\right)$. Introduce the abstract algebra $\mathcal{S}$ with generators $s_{i j}, i, j=1,2, \ldots, 2 n$ and $s_{i i^{\prime}}^{-1}, i=1,3, \ldots, 2 n-1$ with the defining relations given by (2.35), (2.37), (2.38) and

$$
s_{i i^{\prime}} s_{i i^{\prime}}^{-1}=s_{i i^{\prime}}^{-1} s_{i i^{\prime}}=1, \quad i=1,3, \ldots, 2 n-1 .
$$

We show first that the relations (2.38) allow us to eliminate the generators $s_{i^{\prime} i}$ in the spanning set of monomials.

Lemma 2.7. The algebra $\mathcal{S}$ is spanned by the ordered monomials of the form

$$
\prod_{i=1,3, \ldots, 2 n-1}^{\rightarrow} s_{i 1}^{k_{i 1}} s_{i 2}^{k_{i 2}} \cdots s_{i i^{\prime}}^{k_{i i^{\prime}}} s_{i^{\prime} i^{\prime}}^{k_{i^{\prime} i^{\prime}}} s_{i^{\prime} 1}^{k_{i^{\prime} 1}} \cdots s_{i^{\prime}, i^{\prime}-2}^{k_{i^{\prime}, i^{\prime}-2}}
$$

where the $k_{i i^{\prime}}$ with $i=1,3, \ldots, 2 n-1$ are arbitrary integers while the remaining powers $k_{i j}$ are nonnegative integers.

Proof. Denote by $\mathcal{S}^{\prime}$ the algebra with generators $s_{i j}$ and $s_{i i^{\prime}}^{-1}$ and the defining relations given by (2.35), (2.37) and (2.40). The defining relations imply that for any odd $i$ we have

$$
q^{\delta_{i^{\prime}, k}-\delta_{i k}} s_{i i^{\prime}} s_{k l}=q^{\delta_{i l}-\delta_{i^{\prime}, l}} s_{k l} s_{i i^{\prime}} .
$$

Therefore, by Lemma 2.1 the algebra $\mathcal{S}^{\prime}$ is spanned by the monomials

$$
\prod_{i=1,3, \ldots, 2 n-1}^{\rightarrow} s_{i 1}^{k_{i 1}} s_{i 2}^{k_{i 2}} \cdots s_{i i^{\prime}}^{k_{i i^{\prime}}} s_{i^{\prime} 1}^{k_{i^{\prime} 1}} \cdots s_{i^{\prime}, i^{\prime}-1}^{k_{i^{\prime}, i^{\prime}-1}} s_{i^{\prime} i^{\prime}}^{k_{i^{\prime} i^{\prime}}}
$$

where the integers $k_{i i^{\prime}}$ are allowed to be negative. As we noted in the proof of Lemma 2.1] the generators $s_{i a}$ and $s_{i b}$ can be permuted modulo terms of lower weight; 
see (2.25). Therefore, rearranging the generators appropriately, we conclude that $\mathcal{S}^{\prime}$ is also spanned by the monomials

$$
\prod_{i=1,3, \ldots, 2 n-1}^{\rightarrow} s_{i 1}^{k_{i 1}} s_{i 2}^{k_{i 2}} \cdots s_{i i^{\prime}}^{k_{i i^{\prime}}} s_{i^{\prime}, i^{\prime}-1}^{k_{i^{\prime}, i^{\prime}-1}} s_{i^{\prime} i^{\prime}}^{k_{i^{\prime} i^{\prime}}} s_{i^{\prime} 1}^{k_{i^{\prime} 1}} \cdots s_{i^{\prime}, i^{\prime}-2}^{k_{i^{\prime}, i^{\prime}-2}} .
$$

It is a straightforward calculation to derive from the defining relations that the elements $s_{i^{\prime} i^{\prime}} s_{i i}-q^{2} s_{i^{\prime} i} s_{i i^{\prime}}$ with $i=1,3, \ldots, 2 n-1$ are central in the algebra $\mathcal{S}^{\prime}$. Let $I$ be the ideal of $\mathcal{S}^{\prime}$ generated by the central elements $s_{i^{\prime} i^{\prime}} s_{i i}-q^{2} s_{i^{\prime} i} s_{i i^{\prime}}-q^{3}$ for $i=1,3, \ldots, 2 n-1$. We need to show that the quotient $\mathcal{S}^{\prime} / I$ is spanned by the monomials (2.41). However, the defining relations between the generators $s_{i i}, s_{i i^{\prime}}$, $s_{i^{\prime} i}$ and $s_{i^{\prime} i^{\prime}}$ do not involve any other generators. In order to complete the proof it is therefore sufficient to consider the particular case $n=1$. We shall show that modulo the ideal $I$ generated by the central element $s_{22} s_{11}-q^{2} s_{21} s_{12}-q^{3}$ every element of the algebra $\mathcal{S}^{\prime}$ can be written as a linear combination of monomials of the form $s_{11}^{k_{11}} s_{12}^{k_{12}} s_{22}^{k_{22}}$. Let $s_{11}^{k_{11}} s_{12}^{k_{12}} s_{21}^{k_{21}} s_{22}^{k_{22}}$ be an arbitrary monomial of the form (2.44). We assume $k_{21} \geq 1$ and use induction on $k_{21}$. Modulo the ideal $I$ we have

$$
s_{21} \equiv q^{-2} s_{22} s_{11} s_{12}^{-1}+q s_{12}^{-1} .
$$

Therefore, omitting the ordered monomials with smaller powers $k_{21}$ we have the relation modulo the ideal $I$ :

$$
s_{11}^{k_{11}} s_{12}^{k_{12}} s_{21}^{k_{21}} s_{22}^{k_{22}} \equiv q^{-2} s_{11}^{k_{11}} s_{12}^{k_{12}-1} s_{22} s_{11} s_{21}^{k_{21}-1} s_{22}^{k_{22}} .
$$

Note that by (2.22) we have the relation

$$
s_{22} s_{11}=s_{11} s_{22}+\left(q-q^{-1}\right)\left(s_{12}^{2}+q s_{12} s_{21}\right) .
$$

This allows us to bring the right hand side of (2.46) to the form

$$
\left(1-q^{-2}\right) s_{11}^{k_{11}} s_{12}^{k_{12}} s_{21}^{k_{21}} s_{22}^{k_{22}}
$$

which completes the proof.

The following is an analog of Theorem 2.2 for the symplectic case.

Theorem 2.8. The algebra $\mathcal{S}$ is isomorphic to $\mathrm{U}_{q}^{\text {tw }}\left(\mathfrak{s p}_{2 n}\right)$.

Proof. We use the same argument as for the proof of Theorem 2.2. For this proof only, denote the matrix $T G \bar{T}^{t}$ by $\widetilde{S}$ and its matrix elements by $\widetilde{s}_{i j}$. The map $S \mapsto \widetilde{S}$ defines an algebra homomorphism $\varphi: \mathcal{S} \rightarrow \mathrm{U}_{q}^{\text {tw }}\left(\mathfrak{s p}_{2 n}\right)$. We show that the images of the monomials (2.41) under $\varphi$ are linearly independent. Note that by (2.42) the 
product of a monomial of the form (2.41) and $s_{i i^{\prime}}^{k}$ is, up to a nonzero factor, equal to the same monomial with the index $k_{i i^{\prime}}$ replaced with $k_{i i^{\prime}}+k$. Therefore we may assume without loss of generality that all powers in (2.41) are nonnegative integers.

Set $V=\mathrm{U}_{q}^{\mathrm{tw}}\left(\mathfrak{s p}_{2 n}\right)$. The corresponding generators $\sigma_{i j} \in V_{\mathcal{A}}$ can now be given by

$$
\sigma_{i j}=\frac{\widetilde{s}_{i j}-g_{i j}}{q-q^{-1}}
$$

where the $g_{i j}$ are the matrix elements of $G$. We have the relation modulo $(q-1)$,

$$
\sigma_{i j} \equiv \widetilde{g}_{j^{\prime} j} \tau_{i j^{\prime}}+\widetilde{g}_{i i^{\prime}} \bar{\tau}_{j i^{\prime}}
$$

where $\widetilde{g}_{i j}$ is the value of $g_{i j}$ at $q=1$ so that $\widetilde{g}_{i i^{\prime}}=(-1)^{i-1}$. Therefore, the image of the element $\sigma_{i j}$ in $V_{\mathcal{A}} \otimes_{\mathcal{A}} \mathbb{C}$ is $F_{i j}:=\widetilde{g}_{j^{\prime} j} E_{i j^{\prime}}-\widetilde{g}_{i i^{\prime}} E_{j i^{\prime}}$. The elements $F_{i j}$ span a subalgebra of $\mathfrak{g l}_{2 n}$ isomorphic to the symplectic Lie algebra $\mathfrak{s p}_{2 n}$ and this allows us to complete the proof exactly as in the orthogonal case.

The following is an analog of the Poincaré-Birkhoff-Witt theorem for the algebra $\mathcal{S}$ which is immediate from Theorem 2.8.

Corollary 2.9. The monomials (2.41) constitute a basis of the algebra $\mathcal{S}$.

It follows from the proof of the theorem that the algebra $V_{\mathcal{A}} \otimes_{\mathcal{A}} \mathbb{C}$ coincides with the enveloping algebra $\mathrm{U}\left(\mathfrak{s p}_{2 n}\right)$. Thus, $\mathrm{U}_{q}^{\text {tw }}\left(\mathfrak{s p}_{2 n}\right)$ specializes to $\mathrm{U}\left(\mathfrak{s p}_{2 n}\right)$ as $q \rightarrow 1$. The result also follows from [21, Section 6].

Regarding $\mathrm{U}_{q}^{\mathrm{tw}}\left(\mathfrak{s p}_{2 n}\right)$ as a subalgebra of $\mathrm{U}_{q}\left(\mathfrak{g l}_{2 n}\right)$ we introduce another matrix $\bar{S}$ by

$$
\bar{S}=\bar{T} G T^{t}
$$

Using the same argument as in the orthogonal case (see Section 2.1) we derive the following relations between the matrix elements of $S$ and $\bar{S}$ : for any $i=1,3, \ldots 2 n-1$

$$
\begin{aligned}
\bar{s}_{i i} & =-q^{-2} s_{i i}, & \bar{s}_{i^{\prime} i^{\prime}} & =-q^{-2} s_{i^{\prime} i^{\prime}}, \\
\bar{s}_{i^{\prime} i} & =-q^{-1} s_{i i^{\prime}}, & \bar{s}_{i i^{\prime}} & =-q^{-1} s_{i^{\prime} i}+\left(1-q^{-2}\right) s_{i i^{\prime}},
\end{aligned}
$$

while for the remaining generators we have

$$
\bar{s}_{i j}=-q^{-1} s_{j i}, \quad i<j, \quad j \neq i^{\prime} .
$$

Thus, the elements $\bar{s}_{i j}$ belong to the subalgebra $\mathrm{U}_{q}^{\text {tw }}\left(\mathfrak{s p}_{2 n}\right)$.

The next proposition is immediate from (2.11); cf. Proposition 2.4. It shows that the subalgebra $\mathrm{U}_{q}^{\mathrm{tw}}\left(\mathfrak{s p}_{2 n}\right)$ is a left coideal of $\mathrm{U}_{q}\left(\mathfrak{g l}_{2 n}\right)$; see [21, Lemma 6.3]. 
Proposition 2.10. The images of the generators of $\mathrm{U}_{q}^{\mathrm{tw}}\left(\mathfrak{s p}_{2 n}\right)$ under the coproduct are given by

$$
\Delta\left(s_{i j}\right)=\sum_{k, l=1}^{2 n} t_{i k} \bar{t}_{j l} \otimes s_{k l}
$$

and

$$
\Delta\left(s_{i i^{\prime}}^{-1}\right)=t_{i^{\prime} i^{\prime}} \bar{t}_{i i} \otimes s_{i i^{\prime}}^{-1} .
$$

\section{Coideal subalgebras of $\mathrm{U}_{q}\left(\widehat{\mathfrak{g l}}_{N}\right)$}

Consider the Lie algebra of Laurent polynomials $\mathfrak{g l}_{N}\left[\lambda, \lambda^{-1}\right]$ in an indeterminate $\lambda$. We denote it by $\widehat{\mathfrak{g l}}_{N}$ for brevity. The quantum affine algebra $\mathrm{U}_{q}\left(\widehat{\mathfrak{g l}}_{N}\right)$ is a deformation of the universal enveloping algebra $\mathrm{U}\left(\widehat{\mathfrak{g l}}_{N}\right)$. We use its $R$-matrix presentation following Reshetikhin, Takhtajan and Faddeev [36; ;f. Section 2. Note also a recent work of Frenkel and Mukhin [11, where different realizations of $\mathrm{U}_{q}\left(\widehat{\mathfrak{g l}}_{N}\right)$ are collected. By definition, the algebra $\mathrm{U}_{q}\left(\widehat{\mathfrak{g l}}_{N}\right)$ has countably many generators $t_{i j}^{(r)}$ and $\bar{t}_{i j}^{(r)}$ where $1 \leq i, j \leq N$ and $r$ runs over nonnegative integers. They are combined into the matrices

$$
T(u)=\sum_{i, j=1}^{N} t_{i j}(u) \otimes E_{i j}, \quad \bar{T}(u)=\sum_{i, j=1}^{N} \bar{t}_{i j}(u) \otimes E_{i j},
$$

where $t_{i j}(u)$ and $\bar{t}_{i j}(u)$ are formal series in $u^{-1}$ and $u$, respectively:

$$
t_{i j}(u)=\sum_{r=0}^{\infty} t_{i j}^{(r)} u^{-r}, \quad \bar{t}_{i j}(u)=\sum_{r=0}^{\infty} \bar{t}_{i j}^{(r)} u^{r} .
$$

The defining relations are

$$
\begin{aligned}
t_{i j}^{(0)} & =\bar{t}_{j i}^{(0)}=0, \quad 1 \leq i<j \leq N, \\
t_{i i}^{(0)} \bar{t}_{i i}^{(0)} & =\bar{t}_{i i}^{(0)} t_{i i}^{(0)}=1, \quad 1 \leq i \leq N, \\
R(u, v) T_{1}(u) T_{2}(v) & =T_{2}(v) T_{1}(u) R(u, v), \\
R(u, v) \bar{T}_{1}(u) \bar{T}_{2}(v) & =\bar{T}_{2}(v) \bar{T}_{1}(u) R(u, v), \\
R(u, v) \bar{T}_{1}(u) T_{2}(v) & =T_{2}(v) \bar{T}_{1}(u) R(u, v),
\end{aligned}
$$

where we have used the notation of (2.3) and $R(u, v)$ is the trigonometric $R$-matrix given by

$$
\begin{aligned}
R(u, v) & =(u-v) \sum_{i \neq j} E_{i i} \otimes E_{j j}+\left(q^{-1} u-q v\right) \sum_{i} E_{i i} \otimes E_{i i} \\
& +\left(q^{-1}-q\right) u \sum_{i>j} E_{i j} \otimes E_{j i}+\left(q^{-1}-q\right) v \sum_{i<j} E_{i j} \otimes E_{j i} .
\end{aligned}
$$


It satisfies the Yang-Baxter equation

$$
R_{12}(u, v) R_{13}(u, w) R_{23}(v, w)=R_{23}(v, w) R_{13}(u, w) R_{12}(u, v)
$$

where both sides take values in End $\mathbb{C}^{N} \otimes$ End $\mathbb{C}^{N} \otimes$ End $\mathbb{C}^{N}$ and the subindices indicate the copies of End $\mathbb{C}^{N}$, e.g., $R_{12}(u, v)=R(u, v) \otimes 1$ etc. Note that $R(u, v)$ is related with the constant $R$-matrices (2.1) and (2.7) by the formula

$$
R(u, v)=u \widetilde{R}-v R
$$

There is a Hopf algebra structure on $\mathrm{U}_{q}\left(\widehat{\mathfrak{g l}}_{N}\right)$ with the coproduct defined by

$$
\Delta\left(t_{i j}(u)\right)=\sum_{k=1}^{N} t_{i k}(u) \otimes t_{k j}(u), \quad \Delta\left(\bar{t}_{i j}(u)\right)=\sum_{k=1}^{N} \bar{t}_{i k}(u) \otimes \bar{t}_{k j}(u) .
$$

The algebra $\mathrm{U}_{q}\left(\widehat{\mathfrak{g l}}_{N}\right)$ specializes to $\mathrm{U}\left(\widehat{\mathfrak{g l}}_{N}\right)$ as $q \rightarrow 1$. More precisely, as with the case of $\mathrm{U}_{q}\left(\mathfrak{g l}_{N}\right)$ (see Section 2), regard $q$ as a formal variable and introduce the $\mathcal{A}$-subalgebra $\mathrm{U}_{\mathcal{A}}$ of $\mathrm{U}_{q}\left(\widehat{\mathfrak{g l}}_{N}\right)$ generated by the elements $\tau_{i j}^{(r)}$ and $\bar{\tau}_{i j}^{(r)}$ defined by

$$
\tau_{i j}^{(r)}=\frac{t_{i j}^{(r)}}{q-q^{-1}}, \quad \bar{\tau}_{i j}^{(r)}=\frac{\bar{t}_{i j}^{(r)}}{q-q^{-1}}
$$

for $r \geq 0$ and all $i, j$, except for the case $r=0$ and $i=j$ where we set

$$
\tau_{i i}^{(0)}=\frac{t_{i i}^{(0)}-1}{q-1}, \quad \bar{\tau}_{i i}^{(0)}=\frac{\bar{t}_{i i}^{(0)}-1}{q-1} .
$$

Then we have an isomorphism

$$
\mathrm{U}_{\mathcal{A}} \otimes_{\mathcal{A}} \mathbb{C} \cong \mathrm{U}\left(\widehat{\mathfrak{g l}}_{N}\right)
$$

see [2, Section 12.2] and [11, Section 2]. The images of the generators of $\mathrm{U}_{\mathcal{A}}$ in (3.10) are given by

$$
\tau_{i j}^{(r)} \rightarrow E_{i j} \lambda^{r}, \quad \bar{\tau}_{i j}^{(r)} \rightarrow-E_{i j} \lambda^{-r}
$$

for all $r \geq 0$ with the exception $\tau_{i j}^{(0)}=\bar{\tau}_{j i}^{(0)}=0$ if $i<j$. Given a subalgebra $V$ of $\mathrm{U}_{q}\left(\widehat{\mathfrak{g l}}_{N}\right)$ we set $V_{\mathcal{A}}=V \cap \mathrm{U}_{\mathcal{A}}$. We shall say that $V$ specializes to a subalgebra $V^{\circ}$ of $\mathrm{U}\left(\widehat{\mathfrak{g l}}_{N}\right)$ if $V_{\mathcal{A}} \otimes_{\mathcal{A}} \mathbb{C} \cong V^{\circ}$.

The quantized enveloping algebra $\mathrm{U}_{q}\left(\mathfrak{g l}_{N}\right)$ is a natural (Hopf) subalgebra of $\mathrm{U}_{q}\left(\widehat{\mathfrak{g l}}_{N}\right)$ defined by the embedding

$$
t_{i j} \mapsto t_{i j}^{(0)}, \quad \bar{t}_{i j} \mapsto \bar{t}_{i j}^{(0)}
$$


Moreover, there is an algebra homomorphism $\mathrm{U}_{q}\left(\widehat{\mathfrak{g l}}_{N}\right) \rightarrow \mathrm{U}_{q}\left(\mathfrak{g l}_{N}\right)$ called the evaluation homomorphism defined by

$$
T(u) \mapsto T-\bar{T} u^{-1}, \quad \bar{T}(u) \mapsto \bar{T}-T u
$$

The $A$ type quantum affine algebras are exceptional in the sense that only in this case such an evaluation homomorphism does exist; see Chari-Pressley [2, Chapter 12].

The subalgebra of $\mathrm{U}_{q}\left(\widehat{\mathfrak{g l}}_{N}\right)$ generated by the elements $t_{i j}^{(r)}$ was studied e.g. in [3], [30], 36]. We call it the $q$-Yangian. In what follows we construct quantum affine algebras associated with the orthogonal and symplectic Lie algebras for which analogs of the evaluation homomorphism (3.13) do exist; cf. the $B$ and $C$ type twisted Yangians [35], 28]. These algebras can be viewed as twisted analogs of the $q$-Yangian as well as $q$-analogs of the twisted Yangians. Note, however, that contrary to the case of the twisted Yangians, our algebras are not subalgebras of the $q$-Yangian; they are generated by certain combinations of both types of elements $t_{i j}^{(r)}$ and $\bar{t}_{i j}^{(r)}$.

\subsection{Orthogonal twisted $q$-Yangians}

Definition 3.1. The twisted $q$-Yangian $\mathrm{Y}_{q}^{\mathrm{tw}}\left(\mathfrak{o}_{N}\right)$ is the subalgebra of $\mathrm{U}_{q}\left(\widehat{\mathfrak{g l}}_{N}\right)$ generated by the coefficients $s_{i j}^{(r)}$ of the matrix elements of the matrix $S(u)=T(u) \bar{T}\left(u^{-1}\right)^{t}$. More precisely, we have

$$
s_{i j}(u)=\sum_{a=1}^{N} t_{i a}(u) \bar{t}_{j a}\left(u^{-1}\right)
$$

so that

$$
s_{i j}(u)=\sum_{r=0}^{\infty} s_{i j}^{(r)} u^{-r} .
$$

The subalgebra $\mathrm{Y}_{q}^{\mathrm{tw}}\left(\mathfrak{o}_{N}\right)$ is generated by the elements $s_{i j}^{(r)}$ with $1 \leq i, j \leq N$ and $r$ running over the set of nonnegative integers.

Next we give a presentation of the algebra $\mathrm{Y}_{q}^{\mathrm{tw}}\left(\mathfrak{o}_{N}\right)$ in terms of generators and defining relations by analogy with the finite-dimensional case; see Section 2.1. Consider the element $R^{t}(u, v):=R^{t_{1}}(u, v)$ obtained from $R(u, v)$ by the transposition in the first factor:

$$
\begin{aligned}
R^{t}(u, v) & =(u-v) \sum_{i \neq j} E_{i i} \otimes E_{j j}+\left(q^{-1} u-q v\right) \sum_{i} E_{i i} \otimes E_{i i} \\
& +\left(q^{-1}-q\right) u \sum_{i>j} E_{j i} \otimes E_{j i}+\left(q^{-1}-q\right) v \sum_{i<j} E_{j i} \otimes E_{j i} .
\end{aligned}
$$


The following relations are implied by (3.3):

$$
\begin{aligned}
s_{i j}^{(0)} & =0, \quad 1 \leq i<j \leq N, \\
s_{i i}^{(0)} & =1, \quad 1 \leq i \leq N, \\
R(u, v) S_{1}(u) R^{t}\left(u^{-1}, v\right) S_{2}(v) & =S_{2}(v) R^{t}\left(u^{-1}, v\right) S_{1}(u) R(u, v) .
\end{aligned}
$$

We shall be proving that these are precisely the defining relations for the algebra $\mathrm{Y}_{q}^{\mathrm{tw}}\left(\mathfrak{o}_{N}\right)$.

Lemma 3.2. Consider the (abstract) associative algebra with generators $s_{i j}^{(r)}$ where $i, j=1, \ldots, N$ and $r=0,1, \ldots$ The defining relations are written in terms of the matrix $S(u)=\left(s_{i j}(u)\right)$ by the relation (3.19) with $s_{i j}(u)$ defined by (3.15). Introduce the ordering on the generators in such a way that $s_{i j}^{(r)} \preceq s_{k l}^{(p)}$ if and only if $(r, i, j) \preceq$ $(p, k, l)$ in the lexicographical order. Then the ordered monomials in the generators span the algebra.

Proof. Write the defining relations in terms of the generating series $s_{i j}(u)$ :

$$
\begin{aligned}
& \left(q^{-\delta_{i j}} u-q^{\delta_{i j}} v\right) \alpha_{i j a b}(u, v)+\left(q^{-1}-q\right)\left(u \delta_{j<i}+v \delta_{i<j}\right) \alpha_{j i a b}(u, v) \\
= & \left(q^{-\delta_{a b}} u-q^{\delta_{a b}} v\right) \alpha_{j i b a}(v, u)+\left(q^{-1}-q\right)\left(u \delta_{a<b}+v \delta_{b<a}\right) \alpha_{j i a b}(v, u),
\end{aligned}
$$

where we have used the notation

$$
\alpha_{i j a b}(u, v)=\left(q^{-\delta_{a j}}-q^{\delta_{a j}} u v\right) s_{i a}(u) s_{j b}(v)+\left(q^{-1}-q\right)\left(\delta_{j<a}+u v \delta_{a<j}\right) s_{i j}(u) s_{a b}(v)
$$

We shall show that any monomial

$$
s_{i_{1} a_{1}}^{\left(k_{1}\right)} s_{i_{2} a_{2}}^{\left(k_{2}\right)} \cdots s_{i_{p} a_{p}}^{\left(k_{p}\right)}
$$

can be written as a linear combination of the ordered monomials. Define the degree of the monomial (3.22) as the sum $k_{1}+\cdots+k_{p}$ and argue by induction on the degree. The induction base is Lemma 2.1 which takes care of the monomials of degree zero. In other words, we can introduce the filtration on the algebra by setting $\operatorname{deg} s_{i a}^{(k)}=k$ and it will be sufficient to prove the lemma for the corresponding graded algebra. We keep the same notation for its generators while the defining relations are given by (3.20) where instead of (3.21) we should take

$$
\alpha_{i j a b}(u, v)=-q^{\delta_{a j}} s_{i a}(u) s_{j b}(v)+\left(q^{-1}-q\right) \delta_{a<j} s_{i j}(u) s_{a b}(v) .
$$

Furthermore, we apply the same argument to this new algebra considering the filtration defined by setting the degree of $s_{i a}^{(k)}$ to be equal to $i$. The corresponding graded 
algebra is generated by elements $s_{i a}^{(k)}$ with the defining relations (3.20) where the expression $\alpha_{i j a b}(u, v)$ further simplifies to

$$
\alpha_{i j a b}(u, v)=-q^{\delta_{a j}} s_{i a}(u) s_{j b}(v) .
$$

Working with this algebra we have for $i>j$

$$
\begin{aligned}
q^{\delta_{a j}} s_{i a}(u) s_{j b}(v) & =\frac{q^{-\delta_{a b}} u-q^{\delta_{a b}} v}{u-v} q^{\delta_{b i}} s_{j b}(v) s_{i a}(u) \\
& +\frac{q-q^{-1}}{u-v} q^{\delta_{a i}}\left(u s_{j a}(u) s_{i b}(v)-\left(u \delta_{a<b}+v \delta_{b<a}\right) s_{j a}(v) s_{i b}(u)\right) .
\end{aligned}
$$

This implies that the monomials (3.22) with the condition $i_{1} \leq \cdots \leq i_{p}$ span the algebra. Now we take $i=j$ and $a<b$ in (3.20) with the assumption (3.24) to get

$$
\begin{aligned}
q^{\delta_{b i}} s_{i b}(v) s_{i a}(u) & =\frac{q^{-1} u-q v}{q^{-\delta_{a b}} u-q^{\delta_{a b}} v} q^{\delta_{a i}} s_{i a}(u) s_{i b}(v) \\
& +\frac{q-q^{-1}}{q^{-\delta_{a b} u-q^{\delta_{a b}} v}} q^{\delta_{a i}} u s_{i a}(v) s_{i b}(u) .
\end{aligned}
$$

Therefore, the algebra is spanned by the monomials (3.22) such that $\left(i_{1}, a_{1}\right) \preceq \cdots \preceq$ $\left(i_{p}, a_{p}\right)$ in the lexicographical order. Finally, using again (3.20) we note that

$$
s_{i a}(u) s_{i a}(v)=s_{i a}(v) s_{i a}(u)
$$

which implies $\left[s_{i a}^{(k)}, s_{i a}^{(r)}\right]=0$ for all $k, r$. This completes the proof.

Theorem 3.3. The abstract algebra $\mathcal{S}$ generated by elements $s_{i j}^{(r)}$ with $i, j=1, \ldots, N$ and $r \geq 0$ with the defining relations (3.17)-(3.19) is isomorphic to $\mathrm{Y}_{q}^{\mathrm{tw}}\left(\mathfrak{o}_{N}\right)$.

Proof. We use the argument of the proof of Theorem 2.2 appropriately modified for the affine case. Namely, we check first that the matrix elements of the matrix $T(u) \bar{T}\left(u^{-1}\right)^{t}$ satisfy the defining relations of $\mathcal{S}$ so that we have an algebra homomorphism $\mathcal{S} \rightarrow \mathrm{Y}_{q}^{\mathrm{tw}}\left(\mathfrak{o}_{N}\right)$. To prove its injectivity regard $q$ as a formal variable and set $V=\mathrm{Y}_{q}^{\mathrm{tw}}\left(\mathfrak{o}_{N}\right)$. Note that the algebra $V_{\mathcal{A}}$ is generated by the elements

$$
\sigma_{i j}^{(r)}=\frac{s_{i j}^{(r)}}{q-q^{-1}},
$$

where the condition $i>j$ must hold if $r=0$. The image of $\sigma_{i j}^{(r)}$ in $V_{\mathcal{A}} \otimes_{\mathcal{A}} \mathbb{C}$ is $F_{i j}^{(r)}=E_{i j} \lambda^{r}-E_{j i} \lambda^{-r}$. However, the elements $F_{i j}^{(r)}$ span the fixed point subalgebra $\widehat{\mathfrak{g l}}_{N}^{\theta}$ of $\widehat{\mathfrak{g l}}_{N}$ corresponding to the automorphism

$$
\theta: E_{i j} \lambda^{p} \mapsto-E_{j i} \lambda^{-p}
$$

Thus, Lemma 3.2 and this observation complete the proof. 
Consider the ordering on the generators of $\mathcal{S}$ defined in Lemma 3.2. We shall assume that for the generators $s_{i j}^{(0)}$ the condition $i>j$ holds.

Corollary 3.4. The ordered monomials in the generators constitute a basis of the algebra $\mathcal{S}$.

Corollary 3.5. The subalgebra $\mathrm{Y}_{q}^{\mathrm{tw}}\left(\mathfrak{o}_{N}\right)$ of $\mathrm{U}_{q}\left(\widehat{\mathfrak{g l}}_{N}\right)$ specializes to the universal enveloping algebra $\mathrm{U}\left(\widehat{\mathfrak{g l}}_{N}^{\theta}\right)$ as $q \rightarrow 1$.

The following proposition is immediate from (3.7).

Proposition 3.6. The subalgebra $\mathrm{Y}_{q}^{\mathrm{tw}}\left(\mathfrak{o}_{N}\right)$ of $\mathrm{U}_{q}\left(\widehat{\mathfrak{g l}}_{N}\right)$ is a left coideal so that

$$
\Delta\left(s_{i j}(u)\right)=\sum_{k, l=1}^{N} t_{i k}(u) \bar{t}_{j l}\left(u^{-1}\right) \otimes s_{k l}(u) .
$$

The next proposition allows us to regard $\mathrm{U}_{q}^{\mathrm{tw}}\left(\mathfrak{o}_{N}\right)$ as a subalgebra of $\mathrm{Y}_{q}^{\mathrm{tw}}\left(\mathfrak{o}_{N}\right)$.

Proposition 3.7. The map $s_{i j} \mapsto s_{i j}^{(0)}$ defines an embedding $\mathrm{U}_{q}^{\mathrm{tw}}\left(\mathfrak{o}_{N}\right) \hookrightarrow \mathrm{Y}_{q}^{\mathrm{tw}}\left(\mathfrak{o}_{N}\right)$.

Proof. It is clear from (3.17)-(3.19) that the elements $s_{i j}^{(0)}$ satisfy the defining relations for the $s_{i j}$; see (2.15)-(2.17). Corollary 3.4 ensures that the homomorphism is injective.

The following theorem establishes the existence of the evaluation homomorphisms for the twisted $q$-Yangians. We use notation (2.29). As before, we combine the formal series $s_{i j}(u)$ into the matrix $S(u)$ so that $S(u)$ is a formal power series in $u^{-1}$ with matrix coefficients.

Theorem 3.8. The mapping

$$
S(u) \mapsto S+q^{-1} u^{-1} \bar{S}
$$

defines an algebra homomorphism $\mathrm{Y}_{q}^{\mathrm{tw}}\left(\mathfrak{o}_{N}\right) \rightarrow \mathrm{U}_{q}^{\mathrm{tw}}\left(\mathfrak{o}_{N}\right)$.

Proof. Regarding $\mathrm{Y}_{q}^{\mathrm{tw}}\left(\mathfrak{o}_{N}\right)$ as an abstract algebra we need to verify that the relation (3.19) holds when we substitute the right hand side of (3.31) for $S(u)$. That is, we need to verify

$$
\begin{aligned}
(u \widetilde{R}-v R)\left(q u S_{1}+\bar{S}_{1}\right) & \left(\widetilde{R}^{t}-u v R^{t}\right)\left(q v S_{2}+\bar{S}_{2}\right) \\
& =\left(q v S_{2}+\bar{S}_{2}\right)\left(\widetilde{R}^{t}-u v R^{t}\right)\left(q u S_{1}+\bar{S}_{1}\right)(u \widetilde{R}-v R) .
\end{aligned}
$$


Applying (2.3) and (2.10) we derive the relations

$$
\begin{array}{rlrl}
R S_{1} R^{t} S_{2} & =S_{2} R^{t} S_{1} R, & & \widetilde{R} S_{1} R^{t} S_{2}=S_{2} R^{t} S_{1} \widetilde{R} \\
R \bar{S}_{1} R^{t} S_{2}=S_{2} R^{t} \bar{S}_{1} R, & \widetilde{R} \bar{S}_{1} \widetilde{R}^{t} \bar{S}_{2}=\bar{S}_{2} \widetilde{R}^{t} \bar{S}_{1} \widetilde{R} \\
R \bar{S}_{1} \widetilde{R}^{t} S_{2}=S_{2} \widetilde{R}^{t} \bar{S}_{1} R, & \widetilde{R} S_{1} \widetilde{R}^{t} \bar{S}_{2}=\bar{S}_{2} \widetilde{R}^{t} S_{1} \widetilde{R} \\
R \bar{S}_{1} \widetilde{R}^{t} \bar{S}_{2}=\bar{S}_{2} \widetilde{R}^{t} \bar{S}_{1} R, & \widetilde{R} S_{1} R^{t} \bar{S}_{2}=\bar{S}_{2} R^{t} S_{1} \widetilde{R} .
\end{array}
$$

Expanding the products on the left and right hand sides of (3.32) we see that in order to complete the proof, it suffices to verify the following four relations

$$
\begin{aligned}
& R S_{1} R^{t} \bar{S}_{2}-\widetilde{R} \bar{S}_{1} R^{t} S_{2}=\bar{S}_{2} R^{t} S_{1} R-S_{2} R^{t} \bar{S}_{1} \widetilde{R}, \\
& R S_{1} \widetilde{R}^{t} \bar{S}_{2}-\widetilde{R} \bar{S}_{1} \widetilde{R}^{t} S_{2}=\bar{S}_{2} \widetilde{R}^{t} S_{1} R-S_{2} \widetilde{R}^{t} \bar{S}_{1} \widetilde{R}, \\
& \widetilde{R} S_{1} \widetilde{R}^{t} S_{2}-q^{-2} \widetilde{R} \bar{S}_{1} R^{t} \bar{S}_{2}=S_{2} \widetilde{R}^{t} S_{1} \widetilde{R}-q^{-2} \bar{S}_{2} R^{t} \bar{S}_{1} \widetilde{R}, \\
& R S_{1} \widetilde{R}^{t} S_{2}-q^{-2} R \bar{S}_{1} R^{t} \bar{S}_{2}=S_{2} \widetilde{R}^{t} S_{1} R-q^{-2} \bar{S}_{2} R^{t} \bar{S}_{1} R .
\end{aligned}
$$

Let us prove the first of them. Using (2.8) replace $R$ and $\widetilde{R}$, respectively, with $\widetilde{R}+\left(q-q^{-1}\right) P$ and $R-\left(q-q^{-1}\right) P$. Due to (3.33), we now have to check that

$$
P S_{1} R^{t} \bar{S}_{2}+P \bar{S}_{1} R^{t} S_{2}=\bar{S}_{2} R^{t} S_{1} P+S_{2} R^{t} \bar{S}_{1} P
$$

However, this is obvious if we observe that $P S_{1}=S_{2} P, P \bar{S}_{1}=\bar{S}_{2} P$ and $P R^{t}=$ $R^{t} P$. The proof of the second relation in (3.34) is the same. The third and forth relations are also verified in a way similar to each other so we only consider the third one. Note that by (2.8),

$$
R^{t}=\widetilde{R}^{t}+\left(q-q^{-1}\right) Q, \quad Q=P^{t}=\sum_{i, j=1}^{N} E_{i j} \otimes E_{i j} .
$$

Replacing $R^{t}$ and $\widetilde{R}^{t}$, respectively, with $\widetilde{R}^{t}+\left(q-q^{-1}\right) Q$ and $R^{t}-\left(q-q^{-1}\right) Q$ in the third relation and using again (3.33) we conclude that it is now sufficient to verify that

$$
\widetilde{R} S_{1} Q S_{2}+q^{-2} \widetilde{R} \bar{S}_{1} Q \bar{S}_{2}=S_{2} Q S_{1} \widetilde{R}+q^{-2} \bar{S}_{2} Q \bar{S}_{1} \widetilde{R}
$$

Since $P Q=Q P=Q$ and

$$
P A_{1}=A_{2} P
$$

for any matrix $A$, we have $P \bar{S}_{1} Q \bar{S}_{2}=\bar{S}_{2} Q \bar{S}_{1} P$. Therefore, by (2.8) we may replace (3.37) with

$$
\widetilde{R} S_{1} Q S_{2}+q^{-2} R \bar{S}_{1} Q \bar{S}_{2}=S_{2} Q S_{1} \widetilde{R}+q^{-2} \bar{S}_{2} Q \bar{S}_{1} R
$$


Finally, we have the chain of equalities

$$
\widetilde{R} S_{1} Q=\widetilde{R} T_{1} \bar{T}_{1}^{t} Q=\widetilde{R} T_{1} \bar{T}_{2} Q=\bar{T}_{2} T_{1} \widetilde{R} Q=q^{-1} \bar{T}_{2} T_{1} Q=q^{-1} \bar{T}_{2} T_{2}^{t} Q=q^{-1} \bar{S}_{2} Q,
$$

where we have used (2.10) and the relations $\widetilde{R} Q=Q \widetilde{R}=q^{-1} Q$ together with the observation that $A_{1}^{t} Q=A_{2} Q$ implied by (3.38). Thus, $\widetilde{R} S_{1} Q S_{2}=q^{-1} \bar{S}_{2} Q S_{2}$. A similar argument shows that

$$
R \bar{S}_{1} Q \bar{S}_{2}=q S_{2} Q \bar{S}_{2}, \quad S_{2} Q S_{1} \widetilde{R}=q^{-1} S_{2} Q \bar{S}_{2}, \quad \bar{S}_{2} Q \bar{S}_{1} R=q \bar{S}_{2} Q S_{2}
$$

proving (3.39) and the theorem.

\subsection{Symplectic twisted $q$-Yangians}

As in Section 2.2, we use the matrix $G=\left(g_{i j}\right)$ given by (2.33).

Definition 3.9. The twisted $q$-Yangian $\mathrm{Y}_{q}^{\mathrm{tw}}\left(\mathfrak{s p}_{2 n}\right)$ is defined as the subalgebra of $\mathrm{U}_{q}\left(\widehat{\mathfrak{g l}}_{2 n}\right)$ generated by the coefficients $s_{i j}^{(r)}$ of the matrix elements of the matrix $S(u)=$ $T(u) G \bar{T}\left(u^{-1}\right)^{t}$ and the elements $\left(s_{i i^{\prime}}^{(0)}\right)^{-1}$ with $i=1,3, \ldots, 2 n-1$. More precisely, the matrix elements are given by

$$
s_{i j}(u)=q \sum_{a=1}^{n} t_{i, 2 a-1}(u) \bar{t}_{j, 2 a}\left(u^{-1}\right)-\sum_{a=1}^{n} t_{i, 2 a}(u) \bar{t}_{j, 2 a-1}\left(u^{-1}\right)
$$

so that

$$
s_{i j}(u)=\sum_{r=0}^{\infty} s_{i j}^{(r)} u^{-r} .
$$

Now we give a presentation of the algebra $\mathrm{Y}_{q}^{\mathrm{tw}}\left(\mathfrak{s p}_{2 n}\right)$ in terms of generators and defining relations. We shall use the notation (3.16). Denote by $\mathcal{S}$ the associative algebra with (abstract) generators $s_{i j}^{(r)}$ with $1 \leq i, j \leq 2 n$ and $r \geq 0,\left(s_{i i^{\prime}}^{(0)}\right)^{-1}$ with $i=1,3, \ldots, 2 n-1$ and the following defining relations written with the use of the generating series (3.43) and the matrix $S(u)=\left(s_{i j}(u)\right)$ :

$$
s_{i j}^{(0)}=0 \quad \text { for } \quad i<j \text { with } j \neq i^{\prime} \text {; }
$$

also, for any odd $i=1,3, \ldots, 2 n-1$

$$
\begin{array}{r}
s_{i^{\prime} i^{\prime}}^{(0)} s_{i i}^{(0)}-q^{2} s_{i^{\prime} i}^{(0)} s_{i i^{\prime}}^{(0)}=q^{3}, \\
s_{i i^{\prime}}^{(0)}\left(s_{i i^{\prime}}^{(0)}\right)^{-1}=\left(s_{i i^{\prime}}^{(0)}\right)^{-1} s_{i i^{\prime}}^{(0)}=1 ;
\end{array}
$$

and

$$
R(u, v) S_{1}(u) R^{t}\left(u^{-1}, v\right) S_{2}(v)=S_{2}(v) R^{t}\left(u^{-1}, v\right) S_{1}(u) R(u, v) .
$$


Theorem 3.10. The algebra $\mathcal{S}$ is isomorphic to the twisted q-Yangian $\mathrm{Y}_{q}^{\mathrm{tw}}\left(\mathfrak{s p}_{2 n}\right)$.

Proof. Let us verify first that the matrix $G$ satisfies

$$
R(u, v) G_{1} R^{t}\left(u^{-1}, v\right) G_{2}=G_{2} R^{t}\left(u^{-1}, v\right) G_{1} R(u, v) .
$$

We have to check the four relations

$$
\begin{array}{ll}
R G_{1} R^{t} G_{2}=G_{2} R^{t} G_{1} R, & \widetilde{R} G_{1} R^{t} G_{2}=G_{2} R^{t} G_{1} \widetilde{R}, \\
R G_{1} \widetilde{R}^{t} G_{2}=G_{2} \widetilde{R}^{t} G_{1} R, & \widetilde{R} G_{1} \widetilde{R}^{t} G_{2}=G_{2} \widetilde{R}^{t} G_{1} \widetilde{R} .
\end{array}
$$

Applying (2.37) together with (2.8) and (3.36) we see that it is sufficient to check that $R G_{1} Q G_{2}=G_{2} Q G_{1} R$ which can be done by a direct calculation.

Further, repeating the arguments of the proof of Theorem 3.3 we construct the algebra homomorphism $\mathcal{S} \rightarrow \mathrm{Y}_{q}^{\mathrm{tw}}\left(\mathfrak{s p}_{2 n}\right)$. Next we verify directly that the elements $s_{i^{\prime} i^{\prime}}^{(0)} s_{i i}^{(0)}-q^{2} s_{i^{\prime} i}^{(0)} s_{i i^{\prime}}^{(0)}$ with odd $i$ are central in the algebra $\mathcal{S}$.

Now Lemma 2.7 and Lemma 3.2 imply that the algebra $\mathcal{S}$ is spanned by the ordered monomials in the generators $s_{i j}^{(r)}$. Here the triples $(r, i, j)$ are ordered lexicographically except for the submonomials in the generators $s_{i j}^{(0)}$. These submonomials should have the form (2.41) with the $s_{i j}$ replaced by $s_{i j}^{(0)}$, respectively.

Finally, the linear independence of the ordered monomials is proved in the same way as in Theorem 3.3. Namely, setting $V=\mathrm{Y}_{q}^{\mathrm{tw}}\left(\mathfrak{s p}_{2 n}\right)$ we consider the corresponding elements of the algebra $V_{\mathcal{A}}$ given by

$$
\sigma_{i j}^{(0)}=\frac{s_{i j}^{(0)}-g_{i j}}{q-q^{-1}}, \quad \text { and } \quad \sigma_{i j}^{(r)}=\frac{s_{i j}^{(r)}}{q-q^{-1}} \quad \text { for } \quad r \geq 1,
$$

cf. (2.49). Then we have modulo $(q-1)$

$$
\sigma_{i j}^{(r)} \equiv \widetilde{g}_{j^{\prime} j} \tau_{i j^{\prime}}^{(r)}+\widetilde{g}_{i i^{\prime}} \bar{\tau}_{j i^{\prime}}^{(r)}
$$

where $\widetilde{g}_{i i^{\prime}}=(-1)^{i-1}$; see (2.50). Hence, the image of $\sigma_{i j}^{(r)}$ in $V_{\mathcal{A}} \otimes_{\mathcal{A}} \mathbb{C}$ is

$$
F_{i j}^{(r)}:=\widetilde{g}_{j^{\prime} j} E_{i j^{\prime}} \lambda^{r}-\widetilde{g}_{i i^{\prime}} E_{j i^{\prime}} \lambda^{-r}
$$

The elements $F_{i j}^{(r)}$ span a fixed point subalgebra $\widehat{\mathfrak{g l}}_{2 n}^{\theta}$ of $\widehat{\mathfrak{g l}}_{2 n}$ with respect to the automorphism

$$
\theta: E_{i j} \lambda^{r} \mapsto(-1)^{i+j-1} E_{j^{\prime} i^{\prime}} \lambda^{-r} .
$$

The application of the Poincaré-Birkhoff-Witt theorem to the Lie algebra $\widehat{\mathfrak{g l}}_{2 n}^{\theta}$ completes the proof. 
Consider the ordering on the generators of $\mathcal{S}$ defined in the proof of Theorem 3.10 , Corollary 3.11. The ordered monomials in the generators constitute a basis of the algebra $\mathcal{S}$.

Corollary 3.12. The subalgebra $\mathrm{Y}_{q}^{\mathrm{tw}}\left(\mathfrak{s p}_{2 n}\right)$ of $\mathrm{U}_{q}\left(\widehat{\mathfrak{g l}}_{2 n}\right)$ specializes to the universal enveloping algebra $\mathrm{U}\left(\widehat{\mathfrak{g l}}_{2 n}^{\theta}\right)$ as $q \rightarrow 1$.

The following proposition is immediate from (3.7).

Proposition 3.13. The subalgebra $\mathrm{Y}_{q}^{\mathrm{tw}}\left(\mathfrak{s p}_{2 n}\right)$ of $\mathrm{U}_{q}\left(\widehat{\mathfrak{g l}}_{2 n}\right)$ is a left coideal so that

$$
\Delta\left(s_{i j}(u)\right)=\sum_{k, l=1}^{2 n} t_{i k}(u) \bar{t}_{j l}\left(u^{-1}\right) \otimes s_{k l}(u) .
$$

The next proposition allows us to regard $\mathrm{U}_{q}^{\mathrm{tw}}\left(\mathfrak{s p}_{2 n}\right)$ as a subalgebra of $\mathrm{Y}_{q}^{\mathrm{tw}}\left(\mathfrak{s p}_{2 n}\right)$.

Proposition 3.14. The map

$$
s_{i j} \mapsto s_{i j}^{(0)}, \quad s_{i i^{\prime}}^{-1} \mapsto\left(s_{i i^{\prime}}^{(0)}\right)^{-1}
$$

defines an embedding $\mathrm{U}_{q}^{\mathrm{tw}}\left(\mathfrak{s p}_{2 n}\right) \hookrightarrow \mathrm{Y}_{q}^{\mathrm{tw}}\left(\mathfrak{s p}_{2 n}\right)$.

Proof. By the defining relations in $\mathrm{U}_{q}^{\mathrm{tw}}\left(\mathfrak{s p}_{2 n}\right)$ and $\mathrm{Y}_{q}^{\mathrm{tw}}\left(\mathfrak{s p}_{2 n}\right)$ the mapping is clearly an algebra homomorphism. Corollary 3.11 ensures that the homomorphism is injective.

The following is an analog of Theorem 3.8 for the symplectic case. We use notation (2.51).

Theorem 3.15. The mapping

$$
S(u) \mapsto S+q u^{-1} \bar{S}, \quad\left(s_{i i^{\prime}}^{(0)}\right)^{-1} \mapsto s_{i i^{\prime}}^{-1}
$$

defines an algebra homomorphism $\mathrm{Y}_{q}^{\mathrm{tw}}\left(\mathfrak{s p}_{2 n}\right) \rightarrow \mathrm{U}_{q}^{\mathrm{tw}}\left(\mathfrak{s p}_{2 n}\right)$.

Proof. By analogy with the orthogonal case (see Theorem 3.8), we need to verify

$$
\begin{aligned}
(u \widetilde{R}-v R)\left(u S_{1}+q \bar{S}_{1}\right) & \left(\widetilde{R}^{t}-u v R^{t}\right)\left(v S_{2}+q \bar{S}_{2}\right) \\
& =\left(v S_{2}+q \bar{S}_{2}\right)\left(\widetilde{R}^{t}-u v R^{t}\right)\left(u S_{1}+q \bar{S}_{1}\right)(u \widetilde{R}-v R) .
\end{aligned}
$$


Note that (3.33) and the first two relations in (3.34) still hold in the same form for the symplectic case. To prove the theorem we shall verify that the third and fourth relations in (3.34) are respectively replaced by

$$
\begin{aligned}
& \widetilde{R} S_{1} \widetilde{R}^{t} S_{2}-q^{2} \widetilde{R} \bar{S}_{1} R^{t} \bar{S}_{2}=S_{2} \widetilde{R}^{t} S_{1} \widetilde{R}-q^{2} \bar{S}_{2} R^{t} \bar{S}_{1} \widetilde{R} \\
& R S_{1} \widetilde{R}^{t} S_{2}-q^{2} R \bar{S}_{1} R^{t} \bar{S}_{2}=S_{2} \widetilde{R}^{t} S_{1} R-q^{2} \bar{S}_{2} R^{t} \bar{S}_{1} R .
\end{aligned}
$$

The chain of equalities (3.40) is replaced with

$$
\begin{array}{r}
\widetilde{R} S_{1} Q=\widetilde{R} T_{1} G_{1} \bar{T}_{1}^{t} Q=\widetilde{R} T_{1} \bar{T}_{2} G_{1} Q=\bar{T}_{2} T_{1} \widetilde{R} G_{1} Q \\
=-q \bar{T}_{2} T_{1} G_{2} Q=-q \bar{T}_{2} G_{2} T_{2}^{t} Q=-q \bar{S}_{2} Q
\end{array}
$$

where we have used (2.10) and the relation $\widetilde{R} G_{1} Q=-q G_{2} Q$ which is verified directly. Thus, $\widetilde{R} S_{1} Q S_{2}=-q \bar{S}_{2} Q S_{2}$. A similar argument shows that

$$
R \bar{S}_{1} Q \bar{S}_{2}=-q^{-1} S_{2} Q \bar{S}_{2}, \quad S_{2} Q S_{1} \widetilde{R}=-q S_{2} Q \bar{S}_{2}, \quad \bar{S}_{2} Q \bar{S}_{1} R=-q^{-1} \bar{S}_{2} Q S_{2}
$$

completing the proof.

\subsection{Comments on possible variations of the definitions}

- By analogy with the algebras $\mathrm{U}_{q}^{\mathrm{tw}}\left(\mathfrak{o}_{N}\right)$ and $\mathrm{U}_{q}^{\mathrm{tw}}\left(\mathfrak{s p}_{2 n}\right)$ we can introduce the matri$\operatorname{ces} \bar{S}(u)=\left(\bar{s}_{i j}(u)\right)$ by

$$
\bar{S}(u)=\bar{T}(u) T\left(u^{-1}\right)^{t} \quad \text { and } \quad \bar{S}(u)=\bar{T}(u) G T\left(u^{-1}\right)^{t}
$$

in the orthogonal and symplectic case, respectively; cf. Definitions 3.1 and 3.9. Then one can derive the following relations form (3.3):

$$
\begin{aligned}
& \left(u q-u^{-1} q^{-1}\right) \bar{s}_{i j}(u)= \\
& \left(u q^{\delta_{i j}}-u^{-1} q^{-\delta_{i j}}\right) s_{j i}\left(u^{-1}\right)+\left(q-q^{-1}\right)\left(u \delta_{j<i}+u^{-1} \delta_{i<j}\right) s_{i j}\left(u^{-1}\right)
\end{aligned}
$$

in the orthogonal case, and

$$
\begin{aligned}
& \left(u^{-1} q-u q^{-1}\right) \bar{s}_{i j}(u)= \\
& \left(u q^{\delta_{i j}}-u^{-1} q^{-\delta_{i j}}\right) s_{j i}\left(u^{-1}\right)+\left(q-q^{-1}\right)\left(u \delta_{i<j}+u^{-1} \delta_{j<i}\right) s_{i j}\left(u^{-1}\right)
\end{aligned}
$$

in the symplectic case. This implies that the coefficients of the series $\bar{s}_{i j}(u)$ generate the same subalgebra $\mathrm{Y}_{q}^{\mathrm{tw}}\left(\mathfrak{o}_{N}\right)$ or $\mathrm{Y}_{q}^{\mathrm{tw}}\left(\mathfrak{s p}_{2 n}\right)$ of $\mathrm{U}_{q}\left(\widehat{\mathfrak{g l}}_{N}\right)$.

- The construction of the coideal subalgebras $\mathrm{Y}_{q}^{\mathrm{tw}}\left(\mathfrak{o}_{N}\right)$ and $\mathrm{Y}_{q}^{\mathrm{tw}}\left(\mathfrak{s p}_{2 n}\right)$ can be generalized to the case of the centrally extended quantum affine algebra $\mathrm{U}_{q}\left(\widehat{\mathfrak{g l}}_{N}\right)_{c}$. We use 
its presentation given in $[8]$. The $R$-matrix $R(u)$ we employ here is related to $R(u, v)$ by

$$
\left(u q^{-1}-q\right) R(u)=R(u, 1) .
$$

The defining relations (3.3) are then replaced by

$$
\begin{aligned}
R(u / v) T_{1}(u) T_{2}(v) & =T_{2}(v) T_{1}(u) R(u / v), \\
R(u / v) \bar{T}_{1}(u) \bar{T}_{2}(v) & =\bar{T}_{2}(v) \bar{T}_{1}(u) R(u / v), \\
R\left(u q^{-c} / v\right) \bar{T}_{1}(u) T_{2}(v) & =T_{2}(v) \bar{T}_{1}(u) R\left(u q^{c} / v\right) .
\end{aligned}
$$

The definition of the matrix $S(u)$ is modified to

$$
S(u)=T\left(u q^{-c}\right) \bar{T}\left(u^{-1}\right)^{t} \quad \text { and } \quad S(u)=T\left(u q^{-c}\right) G \bar{T}\left(u^{-1}\right)^{t}
$$

in the orthogonal and symplectic case, respectively. However, one can demonstrate that $S(u)$ still satisfies the same reflection equation (3.19) or (3.46) which does not involve the central charge $c$. The corresponding subalgebra is therefore isomorphic to the twisted $q$-Yangian, as an abstract algebra.

\section{Centers of the twisted $q$-Yangians}

In this section we construct a formal series whose coefficients belong to the center of the twisted $q$-Yangian.

We start by recalling the well-known construction of the quantum determinants for the quantum affine algebra $\mathrm{U}_{q}\left(\widehat{\mathfrak{g l}}_{N}\right)$; see e.g. [3], [19], 36]. We use an approach analogous to the case of the Yangian $\mathrm{Y}\left(\mathfrak{g l}_{N}\right)$ [17], [20]; see also [28] for a detailed exposition.

Let us consider the multiple tensor product $\mathrm{U}_{q}\left(\widehat{\mathfrak{g l}}_{N}\right) \otimes\left(\text { End } \mathbb{C}^{N}\right)^{\otimes r}$ and use the notation of (3.3). Then we have the following corollary of (3.5) and (3.3):

$$
R\left(u_{1}, \ldots, u_{r}\right) T_{1}\left(u_{1}\right) \cdots T_{r}\left(u_{r}\right)=T_{r}\left(u_{r}\right) \cdots T_{1}\left(u_{1}\right) R\left(u_{1}, \ldots, u_{r}\right),
$$

where

$$
R\left(u_{1}, \ldots, u_{r}\right)=\prod_{i<j} R_{i j}\left(u_{i}, u_{j}\right),
$$

with the product taken in the lexicographical order on the pairs $(i, j)$. The proof of (4.1) is exactly the same as for the Yangians; see e.g. [28]. Furthermore, consider the $q$-permutation operator $P^{q} \in \operatorname{End}\left(\mathbb{C}^{N} \otimes \mathbb{C}^{N}\right)$ defined by

$$
P^{q}=\sum_{i} E_{i i} \otimes E_{i i}+q \sum_{i>j} E_{i j} \otimes E_{j i}+q^{-1} \sum_{i<j} E_{i j} \otimes E_{j i} .
$$


The action of symmetric group $\mathfrak{S}_{r}$ on the space $\left(\mathbb{C}^{N}\right)^{\otimes r}$ can be defined by setting $s_{i} \mapsto P_{s_{i}}^{q}:=P_{i, i+1}^{q}$ for $i=1, \ldots, r-1$, where $s_{i}$ denotes the transposition $(i, i+1)$. If $\sigma=s_{i_{1}} \cdots s_{i_{l}}$ is a reduced decomposition of an element $\sigma \in \mathfrak{S}_{r}$ we set $P_{\sigma}^{q}=$ $P_{s_{i_{1}}}^{q} \cdots P_{s_{i_{l}}}^{q}$. We denote by $A_{r}^{q}$ the $q$-antisymmetrizer

$$
A_{r}^{q}=\sum_{\sigma \in \mathfrak{S}_{r}} \operatorname{sgn} \sigma \cdot P_{\sigma}^{q}
$$

The following proposition is proved by induction on $r$ in the same way as for the Yangians [28] with the use of a property of the reduced decompositions [16, p.50].

Proposition 4.1. We have the relation in End $\left(\mathbb{C}^{N}\right)^{\otimes r}$ :

$$
R\left(1, q^{-2}, \ldots, q^{-2 r+2}\right)=\prod_{0 \leq i<j \leq r-1}\left(q^{-2 i}-q^{-2 j}\right) A_{r}^{q} .
$$

Now (4.1) implies

$$
A_{r}^{q} T_{1}(u) \cdots T_{r}\left(q^{-2 r+2} u\right)=T_{r}\left(q^{-2 r+2} u\right) \cdots T_{1}(u) A_{r}^{q}
$$

which equals

$$
\sum_{a_{i}, b_{i}} t_{b_{1} \cdots b_{r}}^{a_{1} \cdots a_{r}}(u) \otimes E_{a_{1} b_{1}} \otimes \cdots \otimes E_{a_{r} b_{r}}
$$

for some elements $t_{b_{1} \cdots b_{r}}^{a_{1} \cdots a_{r}}(u)$ of $\mathrm{U}_{q}\left(\widehat{\mathfrak{g l}}_{N}\right)\left[\left[u^{-1}\right]\right]$ which we call the quantum minors. They can be given by the following formulas which are immediate from the definition. If $a_{1}<\cdots<a_{r}$ then

$$
t_{b_{1} \cdots b_{r}}^{a_{1} \cdots a_{r}}(u)=\sum_{\sigma \in \mathfrak{S}_{r}}(-q)^{-l(\sigma)} \cdot t_{a_{\sigma(1)} b_{1}}(u) \cdots t_{a_{\sigma(r)} b_{r}}\left(q^{-2 r+2} u\right)
$$

and for any $\tau \in \mathfrak{S}_{r}$ we have

$$
t_{b_{1} \cdots b_{r}}^{a_{\tau(1)} \cdots a_{\tau(r)}}(u)=(-q)^{l(\tau)} t_{b_{1} \cdots b_{r}}^{a_{1} \cdots a_{r}}(u)
$$

where $l(\sigma)$ denotes the length of the permutation $\sigma$. If $b_{1}<\cdots<b_{r}$ (and the $a_{i}$ are arbitrary) then

$$
t_{b_{1} \cdots b_{r}}^{a_{1} \cdots a_{r}}(u)=\sum_{\sigma \in \mathfrak{S}_{r}}(-q)^{l(\sigma)} \cdot t_{a_{r} b_{\sigma(r)}}\left(q^{-2 r+2} u\right) \cdots t_{a_{1} b_{\sigma(1)}}(u)
$$

and for any $\tau \in \mathfrak{S}_{r}$ we have

$$
t_{b_{\tau(1)} \cdots b_{\tau(r)}}^{a_{1} \cdots a_{r}}(u)=(-q)^{-l(\tau)} t_{b_{1} \cdots b_{r}}^{a_{1} \cdots a_{r}}(u)
$$


Moreover, the quantum minor is zero if two top or two bottom indices are equal. Note also that the standard row and column expansion formulas can be easily derived from (4.8) or (4.10). In particular, we have

$$
t_{b_{1} \cdots b_{r}}^{a_{1} \cdots a_{r}}(u)=\sum_{l=1}^{r}(-q)^{-l+1} t_{a_{l} b_{1}}(u) t_{b_{2} \cdots b_{r}}^{a_{1} \cdots \widehat{a}_{l} \cdots a_{r}}\left(q^{-2} u\right)
$$

where the hat indicates the index to be omitted.

The quantum minors $\bar{t}_{b_{1} \cdots b_{r}}^{a_{1} \cdots a_{r}}(u)$ of the matrix $\bar{T}(u)$ are given by the same formulas where the $t_{i j}(u)$ are respectively replaced with $\bar{t}_{i j}(u)$. Furthermore, for any indices $i, j$ we have the well known relations which are deduced from (4.1):

$$
\left[t_{c_{i} d_{j}}(u), t_{d_{1} \cdots d_{r}}^{c_{1} \cdots c_{r}}(v)\right]=0, \quad\left[t_{c_{i} d_{j}}(u), \bar{t}_{d_{1} \cdots d_{r}}^{c_{1} \cdots c_{r}}(v)\right]=0
$$

and the same holds with $t_{c_{i} d_{j}}(u)$ replaced by $\bar{t}_{c_{i} d_{j}}(u)$. For their proof introduce an extra copy of End $\mathbb{C}^{N}$ as a tensor factor which will be enumerated by the index 0 . Now we specialize the parameters $u_{i}$ in (4.1) as follows:

$$
u_{0}=v, \quad u_{i}=q^{-2 i+2} u \quad \text { for } i=1, \ldots, r .
$$

Then by Proposition 4.1 the element (4.2) will take the form

$$
R\left(v, u, \ldots, q^{-2 r+2} u\right)=\prod_{i=1}^{r} R_{0 i}\left(v, q^{-2 i+2} u\right) A_{r}^{q}
$$

Using the definition of the quantum minors and equating the matrix elements on both sides of (4.1) we get the first relation in (4.13); cf. 28]. The proof of the second is similar.

The quantum determinants of the matrices $T(u)$ and $\bar{T}(u)$ are respectively defined by the relations

$$
q \operatorname{det} T(u)=t_{1 \cdots N}^{1 \cdots N}(u), \quad q \operatorname{det} \bar{T}(u)=\bar{t}_{1 \cdots N}^{1 \cdots N}(u)
$$

Write

$$
\operatorname{qdet} T(u)=\sum_{k=0}^{\infty} d_{k} u^{-k}, \quad \text { qdet } \bar{T}(u)=\sum_{k=0}^{\infty} \bar{d}_{k} u^{k}, \quad d_{k}, \bar{d}_{k} \in \mathrm{U}_{q}\left(\widehat{\mathfrak{g l}}_{N}\right) .
$$

Due to the property (4.13), the coefficients $d_{k}$ and $\bar{d}_{k}$ belong to the center of the algebra $\mathrm{U}_{q}\left(\widehat{\mathfrak{g l}}_{N}\right)$. Note also the relation $d_{0} \bar{d}_{0}=1$ which is implied by (B.3) . 


\subsection{Sklyanin determinant}

We shall consider the orthogonal and symplectic cases simultaneously unless otherwise stated. The symbol $\mathrm{Y}_{q}^{\mathrm{tw}}$ will denote either $\mathrm{Y}_{q}^{\mathrm{tw}}\left(\mathfrak{o}_{N}\right)$ or $\mathrm{Y}_{q}^{\mathrm{tw}}\left(\mathfrak{s p}_{N}\right)$ (the latter with $N=2 n)$. As before, we denote by $S(u)$ the matrix $T(u) G \bar{T}\left(u^{-1}\right)^{t}$, where $G$ is the identity matrix in the orthogonal case, and $G$ is given by (2.33) in the symplectic case. Then $S(u)$ satisfies the relations (3.19) and (3.46), respectively, which have the same form. Our arguments are similar to those used in [35] and 28] for the construction of the Sklyanin determinant for the twisted Yangians.

The following relation in the algebra $\mathrm{Y}_{q}^{\mathrm{tw}} \otimes\left(\text { End } \mathbb{C}^{N}\right)^{\otimes r}$ is a corollary of (3.46) .

$$
\begin{gathered}
R\left(u_{1}, \ldots, u_{r}\right) S_{1}\left(u_{1}\right) R_{12}^{t} \cdots R_{1 r}^{t} S_{2}\left(u_{2}\right) R_{23}^{t} \cdots R_{2 r}^{t} S_{3}\left(u_{3}\right) \cdots R_{r-1, r}^{t} S_{r}\left(u_{r}\right)= \\
S_{r}\left(u_{r}\right) R_{r-1, r}^{t} \cdots S_{3}\left(u_{3}\right) R_{2 r}^{t} \cdots R_{23}^{t} S_{2}\left(u_{2}\right) R_{1 r}^{t} \cdots R_{12}^{t} S_{1}\left(u_{1}\right) R\left(u_{1}, \ldots, u_{r}\right)
\end{gathered}
$$

where $R\left(u_{1}, \ldots, u_{r}\right)$ is given by (4.2) and $R_{i j}^{t}=R_{i j}^{t}\left(u_{i}^{-1}, u_{j}\right)$. Now take $r=N$ and specify $u_{i}=u q^{-2 i+2}$. Then Proposition 4.1 implies

$$
\begin{aligned}
& A_{N}^{q} S_{1}(u) R_{12}^{t} \cdots R_{1 N}^{t} S_{2}\left(u q^{-2}\right) R_{23}^{t} \cdots R_{2 N}^{t} S_{3}\left(u q^{-4}\right) \cdots R_{N-1, N}^{t} S_{N}\left(u q^{-2 N+2}\right)= \\
& S_{N}\left(u q^{-2 N+2}\right) R_{N-1, N}^{t} \cdots S_{3}\left(u q^{-4}\right) R_{2 N}^{t} \cdots R_{23}^{t} S_{2}\left(u q^{-2}\right) R_{1 N}^{t} \cdots R_{12}^{t} S_{1}(u) A_{N}^{q} .
\end{aligned}
$$

Since the $q$-antisymmetrizer $A_{N}^{q}$ is proportional to an idempotent and maps the space $\left(\mathbb{C}^{N}\right)^{\otimes N}$ into a one-dimensional subspace, both sides must be equal to $A_{N}^{q}$ times a series sdet $S(u)$ in $u^{-1}$ with coefficients in $\mathrm{Y}_{q}^{\mathrm{tw}}$. We call this series the Sklyanin determinant.

The following theorem provides an expression of sdet $S(u)$ in terms of the quantum determinants.

Theorem 4.2. We have

$$
\text { sdet } S(u)=\gamma_{N}(u) q \operatorname{det} T(u) q \operatorname{det} \bar{T}\left(q^{2 N-2} u^{-1}\right)
$$

where

$$
\gamma_{N}(u)=\prod_{1 \leq i<j \leq N}\left(q^{2 i-2} u^{-1}-q^{-2 j+2} u\right) \cdot \begin{cases}1 & \text { in the case of } \mathfrak{o}_{N} \\ \frac{q^{n-2}-q^{n} u^{2}}{q^{2 n-2}-q^{-2 n} u^{2}} & \text { in the case of } \mathfrak{s p}_{2 n} .\end{cases}
$$

Proof. We follow the arguments of [28, Section 4]. Substitute $S(u)=T(u) G \bar{T}\left(u^{-1}\right)^{t}$ into (4.19) and transform the left hand side using the relations

$$
\bar{T}_{i}\left(u^{-1}\right)^{t} R_{i j}^{t}\left(u^{-1}, v\right) T_{j}(v)=T_{j}(v) R_{i j}^{t}\left(u^{-1}, v\right) \bar{T}_{i}\left(u^{-1}\right)^{t}
$$


which are implied by (3.3). We then bring it to the form

$$
A_{N}^{q} T_{1}(u) \cdots T_{N}\left(q^{-2 N+2} u\right) \widetilde{R}(u) \bar{T}_{1}\left(u^{-1}\right)^{t} \cdots \bar{T}_{N}\left(q^{2 N-2} u^{-1}\right)^{t},
$$

where

$$
\widetilde{R}(u)=G_{1} R_{12}^{t} \cdots R_{1 N}^{t} G_{2} R_{23}^{t} \cdots R_{2 N}^{t} G_{3} \cdots R_{N-1, N}^{t} G_{N} .
$$

By the definition of the quantum determinant we have

$$
A_{N}^{q} T_{1}(u) \cdots T_{N}\left(q^{-2 N+2} u\right)=A_{N}^{q} \operatorname{qdet} T(u) .
$$

Further, using the homomorphism $S(u) \mapsto G$ we derive from (4.19)

$$
A_{N}^{q} \widetilde{R}(u)=G_{N} R_{N-1, N}^{t} \cdots G_{3} R_{2 N}^{t} \cdots R_{23}^{t} G_{2} R_{1 N}^{t} \cdots R_{12}^{t} G_{1} A_{N}^{q} .
$$

Thus, this expression equals $A_{N}^{q} \gamma_{N}(u)$ for a scalar function $\gamma_{N}(u)$. Using the explicit formulas (4.8) one easily derives that

$$
A_{N}^{q} \bar{T}_{1}\left(u^{-1}\right)^{t} \cdots \bar{T}_{N}\left(q^{2 N-2} u^{-1}\right)^{t}=A_{N}^{q} \operatorname{qdet} \bar{T}\left(q^{2 N-2} u^{-1}\right) .
$$

It remains to calculate the function $\gamma_{N}(u)$. Consider first the orthogonal case. Apply the operator $A_{N}^{q} \widetilde{R}(u)$ to the basis vector $e_{1} \otimes \cdots \otimes e_{N}$, where the $e_{i}$ denote the canonical basis vectors of $\mathbb{C}^{N}$. By (3.16) the result is clearly $\gamma_{N}(u) A_{N}^{q}\left(e_{1} \otimes \cdots \otimes e_{N}\right)$ with $\gamma_{N}(u)$ given by (4.21). In the symplectic case apply $A_{2 n}^{q} \widetilde{R}(u)$ to the basis vector

$$
v=e_{2 n-1} \otimes e_{2 n-3} \otimes \cdots \otimes e_{1} \otimes e_{2} \otimes e_{4} \otimes \cdots \otimes e_{2 n} .
$$

Since $G e_{2 k}=q e_{2 k-1}$ the vector $A_{2 n}^{q} \widetilde{R}(u) v$ equals

$$
q^{n} \prod_{n \leq i<j \leq 2 n}\left(q^{2 i-2} u^{-1}-q^{-2 j+2} u\right) A_{2 n}^{q} G_{1} R_{12}^{t} \cdots R_{1,2 n}^{t} G_{2} \cdots G_{n} R_{n, n+1}^{t} \cdots R_{n, 2 n}^{t} w
$$

where

$$
w=e_{2 n-1} \otimes e_{2 n-3} \otimes \cdots \otimes e_{1} \otimes e_{1} \otimes e_{3} \otimes \cdots \otimes e_{2 n-1} .
$$

If $j>n+1$ then $R_{n, j}^{t} w=\left(q^{2 n-2} u^{-1}-q^{-2 j+2} u\right) w$. Further, we have

$$
R_{n, n+1}^{t}\left(e_{1} \otimes e_{1}\right)=\left(q^{2 n-3} u^{-1}-q^{-2 n+1} u\right)\left(e_{1} \otimes e_{1}\right)+\left(q^{-1}-q\right) q^{-2 n} u \sum_{k=2}^{2 n}\left(e_{k} \otimes e_{k}\right)
$$

Next apply the operator $G_{n}$ and note that due to the subsequent application of the $q$-antisymmetrizer, we may only keep the linear combination of the tensor products 
containing $e_{1}$ or $e_{2}$ on the $n$-th and $(n+1)$-th places (see [28, Section 4] for a similar argument in the symplectic twisted Yangian case). That is, we may write

$$
\begin{aligned}
& A_{2 n}^{q} G_{1} R_{12}^{t} \cdots R_{1,2 n}^{t} G_{2} \cdots G_{n} R_{n, n+1}^{t} w= \\
& \quad\left(q^{2 n-4} u^{-1}-q^{-2 n+2} u\right) A_{2 n}^{q} G_{1} R_{12}^{t} \cdots R_{1,2 n}^{t} G_{2} \cdots G_{n-1} R_{n-1, n}^{t} \cdots R_{n-1,2 n}^{t} w^{\prime}
\end{aligned}
$$

with

$$
w^{\prime}=e_{2 n-1} \otimes e_{2 n-3} \otimes \cdots \otimes e_{3} \otimes e_{1} \otimes e_{2} \otimes e_{3} \otimes \cdots \otimes e_{2 n-1} .
$$

By the skew-symmetry of the antisymmetrizer replace $w^{\prime}$ with the vector

$$
w^{\prime \prime}=e_{2 n-1} \otimes e_{2 n-3} \otimes \cdots \otimes e_{3} \otimes e_{3} \otimes e_{2} \otimes e_{1} \otimes \cdots \otimes e_{2 n-1},
$$

taking the sign into account. Continuing the calculation in a similar manner, we conclude that the product of the scalar factors occurring in the procedure will coincide with $\gamma_{2 n}(u)$ given in (4.21) with $N=2 n$.

The centrality of qdet $T(u)$ and $q \operatorname{det} \bar{T}(u)$ in $\mathrm{U}_{q}\left(\widehat{\mathfrak{g l}}_{N}\right)$ immediately implies the corresponding property of the Sklyanin determinant sdet $S(u)$.

Corollary 4.3. The coefficients of the series sdet $S(u)$ belong to the center of the algebra $\mathrm{Y}_{q}^{\mathrm{tw}}$.

Introduce the series $c(u)$ and the elements $c_{k}$ of the center of the algebra $\mathrm{Y}_{q}^{\mathrm{tw}}$ by the formula

$$
c(u)=\gamma_{N}(u)^{-1} \operatorname{sdet} S(u)=1+\sum_{k=1}^{\infty} c_{k} u^{-k} .
$$

The series begins with 1 due to Theorem 4.2 and the relation $d_{0} \bar{d}_{0}=1$.

Proposition 4.4. The coefficients $c_{k}, k \geq 1$ are algebraically independent.

Proof. The coefficients $\left\{d_{k}, k \geq 0, \bar{d}_{k}, k \geq 1\right\}$ of the quantum determinants are algebraically independent. This can be derived by analogy with the case of the Yangian; see e.g. [28, Section 2]. The key observation here is the isomorphism (3.10). The statement is now implied by Theorem 4.2 .

Since both evaluation homomorphisms (3.31) and (3.55) are surjective, we obtain families of central elements in the algebras $\mathrm{U}_{q}^{\mathrm{tw}}\left(\mathfrak{o}_{N}\right)$ and $\mathrm{U}_{q}^{\mathrm{tw}}\left(\mathfrak{s p}_{2 n}\right)$ as images of the coefficients of sdet $S(u)$. In other words, we have the following result.

Proposition 4.5. The coefficients of the Sklyanin determinants $\operatorname{sdet}\left(S+q^{-1} u^{-1} \bar{S}\right)$ and $\operatorname{sdet}\left(S+q u^{-1} \bar{S}\right)$ are central elements in the algebras $\mathrm{U}_{q}^{\mathrm{tw}}\left(\mathfrak{o}_{N}\right)$ and $\mathrm{U}_{q}^{\mathrm{tw}}\left(\mathfrak{s p}_{2 n}\right)$, respectively. 
In what follows we only consider the case of the orthogonal twisted $q$-Yangian $\mathrm{Y}_{q}^{\mathrm{tw}}\left(\mathfrak{o}_{N}\right)$. In order to produce an explicit formula for the corresponding Sklyanin determinant we introduce a map

$$
\pi_{N}: \mathfrak{S}_{N} \rightarrow \mathfrak{S}_{N}, \quad p \mapsto p^{\prime}
$$

which was previously used in the 'short' formula for the Sklyanin determinant for the twisted Yangians; see [27. This map is defined by an inductive procedure. Given a set of positive integers $\omega_{1}<\cdots<\omega_{N}$ we regard $\mathfrak{S}_{N}$ as the group of their permutations. If $N=2$ we define $\pi_{2}$ as the map $\mathfrak{S}_{2} \rightarrow \mathfrak{S}_{2}$ whose image is the identity permutation. For $N>2$ define a map from the set of ordered pairs $\left(\omega_{k}, \omega_{l}\right)$ with $k \neq l$ into itself by the rule

$$
\begin{aligned}
\left(\omega_{k}, \omega_{l}\right) & \mapsto\left(\omega_{l}, \omega_{k}\right), & & k, l<N, \\
\left(\omega_{k}, \omega_{N}\right) & \mapsto\left(\omega_{N-1}, \omega_{k}\right), & & k<N-1, \\
\left(\omega_{N}, \omega_{k}\right) & \mapsto\left(\omega_{k}, \omega_{N-1}\right), & & k<N-1, \\
\left(\omega_{N-1}, \omega_{N}\right) & \mapsto\left(\omega_{N-1}, \omega_{N-2}\right), & & \\
\left(\omega_{N}, \omega_{N-1}\right) & \mapsto\left(\omega_{N-1}, \omega_{N-2}\right) . & &
\end{aligned}
$$

Let $p=\left(p_{1}, \ldots, p_{N}\right)$ be a permutation of the indices $\omega_{1}, \ldots, \omega_{N}$. Its image under the map $\pi_{N}$ is the permutation $p^{\prime}=\left(p_{1}^{\prime}, \ldots, p_{N-1}^{\prime}, \omega_{N}\right)$, where the pair $\left(p_{1}^{\prime}, p_{N-1}^{\prime}\right)$ is the image of the ordered pair $\left(p_{1}, p_{N}\right)$ under the map (4.37). Then the pair $\left(p_{2}^{\prime}, p_{N-2}^{\prime}\right)$ is found as the image of $\left(p_{2}, p_{N-1}\right)$ under the map (4.37) which is defined on the set of ordered pairs of elements obtained from $\left(\omega_{1}, \ldots, \omega_{N}\right)$ by deleting $p_{1}$ and $p_{N}$; etc. The map $\pi_{N}$ has curious combinatorial properties which were observed by Lascoux; see [26]. In particular, each fiber of this map is an interval in $\mathfrak{S}_{N}$ with respect to the Bruhat order, isomorphic to a Boolean poset.

Example 4.6. The Bruhat order on $\mathfrak{S}_{3}$ and the fibers of the map $\pi_{3}$ :
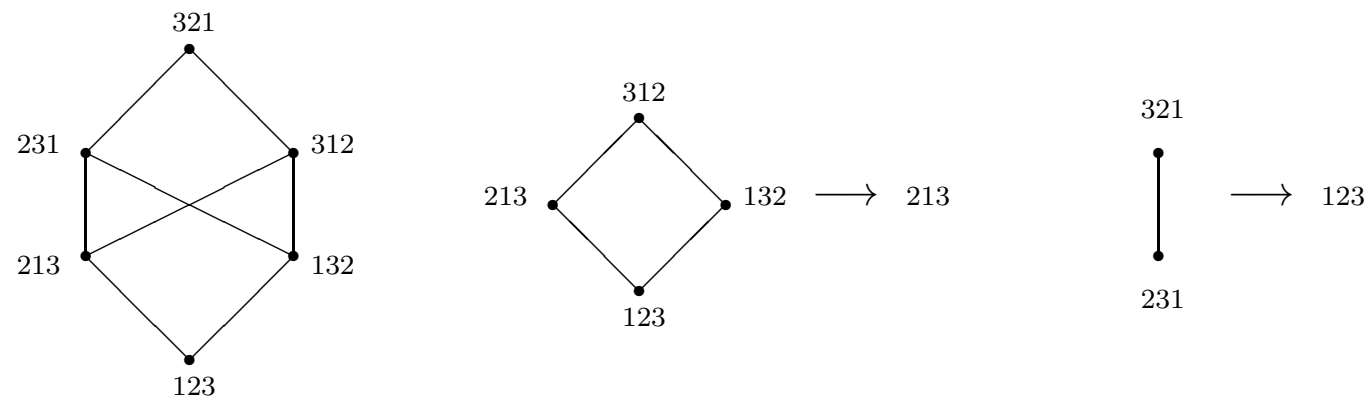

Consider the matrix $\bar{S}(u)$ introduced in (3.60). By (3.61) the matrix elements of $\bar{S}(u)$ are formal series in $u$ with coefficients in the subalgebra $\mathrm{Y}_{q}^{\mathrm{tw}}\left(\mathfrak{o}_{N}\right)$.

Let $n$ denote the rank of the Lie algebra $\mathfrak{o}_{N}$ so that $N=2 n$ or $N=2 n+1$. 
Theorem 4.7. We have an explicit formula

$$
\begin{array}{r}
c(u)=\sum_{p \in \mathfrak{S}_{N}}(-q)^{-l(p)+l\left(p^{\prime}\right)} \bar{s}_{p_{1} p_{1}^{\prime}}^{t}\left(u^{-1}\right) \cdots \bar{s}_{p_{n} p_{n}^{\prime}}^{t}\left(q^{2 n-2} u^{-1}\right) \\
\times s_{p_{n+1} p_{n+1}^{\prime}}\left(q^{-2 n} u\right) \cdots s_{p_{N} p_{N}^{\prime}}\left(q^{-2 N+2} u\right),
\end{array}
$$

where the $\bar{s}_{i j}^{t}(u)$ denote the matrix elements of the transposed matrix $\bar{S}^{t}(u)$.

Example 4.8. If $N=2$ then

$$
c(u)=\bar{s}_{11}^{t}\left(u^{-1}\right) s_{22}\left(q^{-2} u\right)-q^{-1} \bar{s}_{21}^{t}\left(u^{-1}\right) s_{12}\left(q^{-2} u\right) .
$$

If $N=3$ then

$$
\begin{aligned}
c(u) & =\bar{s}_{22}^{t}\left(u^{-1}\right) s_{11}\left(q^{-2} u\right) s_{33}\left(q^{-4} u\right)+\bar{s}_{12}^{t}\left(u^{-1}\right) s_{31}\left(q^{-2} u\right) s_{23}\left(q^{-4} u\right) \\
& +q^{-2} \bar{s}_{21}^{t}\left(u^{-1}\right) s_{32}\left(q^{-2} u\right) s_{13}\left(q^{-4} u\right)-q \bar{s}_{12}^{t}\left(u^{-1}\right) s_{21}\left(q^{-2} u\right) s_{33}\left(q^{-4} u\right) \\
& -q^{-1} \bar{s}_{32}^{t}\left(u^{-1}\right) s_{11}\left(q^{-2} u\right) s_{23}\left(q^{-4} u\right)-q^{-3} \bar{s}_{31}^{t}\left(u^{-1}\right) s_{22}\left(q^{-2} u\right) s_{13}\left(q^{-4} u\right) .
\end{aligned}
$$

Proof of Theorem 4.7. Our proof is based on the properties of the quantum minors of the matrices $T(u)$ and $\bar{T}(u)$. Let $a_{1}, \ldots, a_{r}$ and $b_{1}, \ldots, b_{r}$ be indices from the set $\{1, \ldots, N\}$. Introduce the elements

$$
s_{b_{1} \cdots b_{r}}^{a_{1} \cdots a_{r}}(u)=\sum_{c_{1}<\cdots<c_{r}} t_{c_{1} \cdots c_{r}}^{a_{1} \cdots a_{r}}(u) \bar{t}_{c_{r} \cdots c_{1}}^{b_{r} \cdots b_{1}}\left(q^{2 r-2} u^{-1}\right),
$$

where the indices $c_{1}, \ldots, c_{r}$ run over the set $\{1, \ldots, N\}$. In particular, $s_{b}^{a}(u)=s_{a b}(u)$, and

$$
s_{1 \cdots N}^{1 \cdots N}(u)=c(u)
$$

by (4.20). We shall now derive a recurrent formula for the elements (4.40) with the conditions $b_{i}=a_{i}$ for $i=1, \ldots, r-1$ and $a_{1}<\cdots<a_{r}$. First, by the formulas (4.8) - (4.13) for the quantum minors we can write

$$
\begin{aligned}
r ! s_{a_{1} \cdots a_{r-1}, b_{r}}^{a_{1} \cdots a_{r}}(u) & =\sum_{c_{1}, \ldots, c_{r}} t_{c_{1} \cdots c_{r}}^{a_{1} \cdots a_{r}}(u) \bar{t}_{c_{r} \cdots c_{1}}^{b_{r}, a_{r} \cdots a_{1}}\left(q^{2 r-2} u^{-1}\right) \\
& =r \sum_{c_{1}, \ldots, c_{r}} t_{c_{1} \cdots c_{r}}^{a_{1} \cdots a_{r}}(u) \bar{t}_{c_{r-1} \cdots c_{1}}^{a_{r} \cdots a_{1}}\left(q^{2 r-4} u^{-1}\right) \bar{t}_{b_{r} c_{r}}\left(q^{2 r-2} u^{-1}\right) \\
& =r \sum_{c_{1}, \ldots, c_{r}} \bar{t}_{c_{r-1} \cdots c_{1}}^{a_{r-1} \cdots a_{1}}\left(q^{2 r-4} u^{-1}\right) t_{c_{1} \cdots c_{r}}^{a_{1} \cdots a_{r}}(u) \bar{t}_{b_{r} c_{r}}\left(q^{2 r-2} u^{-1}\right) .
\end{aligned}
$$

Next, applying the column expansion to the quantum minor $t_{c_{1} \cdots c_{r}}^{a_{1} \cdots a_{r}}(u)$, we obtain

$$
\begin{aligned}
& (r-1) ! s_{\substack{a_{1} \cdots a_{r-1}, b_{r} \\
a_{1} \cdots a_{r}}}^{r}(u) \\
& =\sum_{c_{1}, \ldots, c_{r}} \sum_{k=1}^{r}(-q)^{k-r} \bar{t}_{c_{r-1} \cdots c_{1}}^{a_{r-1} \cdots a_{1}}\left(q^{2 r-4} u^{-1}\right) t_{c_{1} \cdots c_{r-1}}^{a_{1} \cdots \widehat{a}_{c_{r} \cdots a_{r}}}(u) t_{a_{k} c_{r}}\left(q^{-2 r+2} u\right) \bar{t}_{b_{r} c_{r}}\left(q^{2 r-2} u^{-1}\right) \\
& =\sum_{c_{1}, \ldots, c_{r-1}} \sum_{k=1}^{r}(-q)^{k-r} \bar{t}_{c_{r-1} \cdots c_{1}}^{a_{r-1} \cdots a_{1}}\left(q^{2 r-4} u^{-1}\right) t_{c_{1} \cdots c_{r-1}}^{a_{1} \cdots \widehat{a}_{k} \cdots a_{r}}(u) s_{a_{k} b_{r}}\left(q^{-2 r+2} u\right),
\end{aligned}
$$


where the hats indicate the symbols to be omitted. Now for any $k<r$ write

$$
\bar{t}_{c_{r-1} \cdots c_{1}}^{a_{r-1} \cdots a_{1}}\left(q^{2 r-4} u^{-1}\right)=(-q)^{k-1} \bar{t}_{c_{r-1} \cdots c_{1}}^{a_{r-1} \cdots \widehat{a}_{1}, a_{k}}\left(q^{2 r-4} u^{-1}\right)
$$

and apply the column expansion to this minor to bring the previous formula to the form

$$
\begin{aligned}
& (r-2) ! s_{a_{1} \cdots a_{r-1}, b_{r}}^{a_{1} \cdots a_{r}}(u) \\
& =\sum_{c_{1}, \ldots, c_{r-1}}\left\{(-q)^{r-2} \bar{t}_{a_{r-1} c_{1}}\left(u^{-1}\right) \bar{t}_{c_{r-1} \cdots c_{2}}^{a_{r-2} \cdots a_{1}}\left(q^{2 r-4} u^{-1}\right) t_{c_{1} \cdots c_{r-1}}^{a_{1} \cdots a_{r-1}}(u) s_{a_{r} b_{r}}\left(q^{-2 r+2} u\right)\right. \\
& \left.+\sum_{k=1}^{r-1}(-q)^{2 k-r-1} \bar{t}_{a_{k} c_{1}}\left(u^{-1}\right) \bar{t}_{c_{r-1} \cdots c_{2}}^{a_{r-1} \cdots \widehat{a}_{k} \cdots a_{1}}\left(q^{2 r-4} u^{-1}\right) t_{c_{1} \cdots c_{r-1}}^{a_{1} \cdots \widehat{a}_{k} \cdots a_{r}}(u) s_{a_{k} b_{r}}\left(q^{-2 r+2} u\right)\right\} .
\end{aligned}
$$

Applying again (4.13), we write this as

$$
\begin{aligned}
& (r-2) ! s_{a_{1} \cdots a_{r-1}, b_{r}}^{a_{1} \cdots a_{r}}(u) \\
& =\sum_{c_{1}, \ldots, c_{r-1}}\left\{(-q)^{r-2} \bar{t}_{a_{r-1} c_{1}}\left(u^{-1}\right) t_{c_{1} \cdots c_{r-1}}^{a_{1} \cdots a_{r-1}}(u) \bar{t}_{c_{r-1} \cdots c_{2}}^{a_{r-2} \cdots a_{1}}\left(q^{2 r-4} u^{-1}\right) s_{a_{r} b_{r}}\left(q^{-2 r+2} u\right)\right. \\
& \left.+\sum_{k=1}^{r-1}(-q)^{2 k-r-1} \bar{t}_{a_{k} c_{1}}\left(u^{-1}\right) t_{c_{1} \cdots c_{r-1}}^{a_{1} \cdots \widehat{a}_{k} \cdots a_{r}}(u) \bar{t}_{c_{r-1} \cdots c_{2}}^{a_{r-1} \cdots \widehat{a}_{k} \cdots a_{1}}\left(q^{2 r-4} u^{-1}\right) s_{a_{k} b_{r}}\left(q^{-2 r+2} u\right)\right\} .
\end{aligned}
$$

Finally, using the column expansion (4.12) and the definition (3.60) of the elements $\bar{s}_{i j}(u)$ we get the following recurrence relation

$$
\begin{aligned}
& s_{a_{1} \cdots a_{r-1}, b_{r}}^{a_{1} \cdots a_{r}}(u)=\bar{s}_{a_{r-1} a_{r-1}}^{t}\left(u^{-1}\right) s_{a_{1} \cdots a_{r-2}}^{a_{1} \cdots a_{r-2}}\left(q^{-2} u\right) s_{a_{r} b_{r}}\left(q^{-2 r+2} u\right) \\
& +\sum_{l=1}^{r-2}(-q)^{2 r-2 l-3} \bar{s}_{a_{l} a_{r-1}}^{t}\left(u^{-1}\right) s_{a_{1} \cdots \widehat{a}_{l} \cdots a_{r-2}, a_{l}}^{a_{1} \cdots \widehat{a}_{l} \cdots a_{r-1}}\left(q^{-2} u\right) s_{a_{r} b_{r}}\left(q^{-2 r+2} u\right) \\
& +\sum_{k=1}^{r-1}\left\{(-q)^{2 k-2 r+1} \bar{s}_{a_{r} a_{k}}^{t}\left(u^{-1}\right) s_{\substack{a_{1} \cdots \widehat{a}_{k} \cdots a_{r-1} \\
a_{1} \cdots a_{r-1}}}\left(q^{-2} u\right) s_{a_{k} b_{r}}\left(q^{-2 r+2} u\right)\right.
\end{aligned}
$$

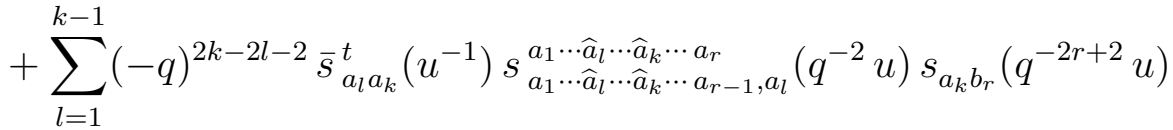

$$
\begin{aligned}
& \left.+\sum_{l=k+1}^{r-1}(-q)^{2 k-2 l} \bar{s}_{a_{l} a_{k}}^{t}\left(u^{-1}\right) s_{a_{1} \cdots \widehat{a}_{k} \cdots \widehat{a}_{l} \cdots a_{r-1}, a_{l}}^{a_{1} \cdots \widehat{a}_{k} \cdots \widehat{a}_{l} \cdots a_{r}}\left(q^{-2} u\right) s_{a_{k} b_{r}}\left(q^{-2 r+2} u\right)\right\} .
\end{aligned}
$$

Using (4.41) and starting with $s_{1 \cdots N}^{1 \cdots N}(u)$ we apply this recurrence relation repeatedly to get an explicit expression for the series $c(u)$ in terms of the generators $s_{i j}(u)$ and $\bar{s}_{i j}(u)$. It follows from the definition of the map $\pi_{N}$ that $c(u)$ will be written as a combination of the monomials of the required form, the coefficients being powers of $-q$. The exact values of the powers are easily found by calculating the number of inversions of the permutations occurring in the recurrence relation. 
Remark 4.9. The argument used in the proof of Theorem 4.7 can also be applied to produce a simpler proof for the formula for the Sklyanin determinant given in [27] in the case of orthogonal and symplectic twisted Yangians. However, the argument does not seem to be directly applicable to the case of the symplectic twisted $q$-Yangian $\mathrm{Y}_{q}^{\mathrm{tw}}\left(\mathfrak{s p}_{2 n}\right)$.

The image of the matrix $\bar{S}(u)$ under the evaluation homomorphism (3.31) is found from the relation (3.61) so that

$$
\bar{S}(u) \mapsto \frac{1+u q^{-1}}{1+u q}(\bar{S}+q u S)
$$

Applying the evaluation homomorphism to the Sklyanin determinant sdet $S(u)$ and using (3.31) and (4.44) we derive the following corollary from Theorem 4.7.

Corollary 4.10. The coefficients of the polynomial

$$
\begin{aligned}
C(u)=\sum_{p \in \mathfrak{S}_{N}}( & -q)^{-l(p)+l\left(p^{\prime}\right)}[u \bar{S}+q S]_{p_{1}^{\prime} p_{1}} \cdots\left[u \bar{S}+q^{2 n-1} S\right]_{p_{n}^{\prime} p_{n}} \\
& \times\left[u S+q^{2 n-1} \bar{S}\right]_{p_{n+1} p_{n+1}^{\prime}} \cdots\left[u S+q^{2 N-3} \bar{S}\right]_{p_{N} p_{N}^{\prime}}
\end{aligned}
$$

are Casimir elements for the algebra $\mathrm{U}_{q}^{\mathrm{tw}}\left(\mathfrak{o}_{N}\right)$. Moreover, the polynomial $C(u)$ is monic of degree $N$.

Proof. The polynomial $C(u)$ is obtained by the application of the evaluation homomorphism to the series $c(u)$ and multiplication by an appropriate rational function in $u$. The centrality of its coefficients thus follows from Theorem 4.7 Obviously, the degree of $C(u)$ does not exceed $N$. The coefficient of $u^{N}$ can only occur in the summands with the property $p=p^{\prime}$. However, it follows from the definition of the map (4.36) that this property is satisfied by the only permutation $p$

$$
p= \begin{cases}(N-1, N-3, \ldots, 1,2,4, \ldots, N) & \text { if } N \quad \text { is even } \\ (N-1, N-3, \ldots, 2,1,3, \ldots, N) & \text { if } N \quad \text { is odd }\end{cases}
$$

Since the diagonal entries of $S$ and $\bar{S}$ are ones, the second statement follows.

The polynomial $C(u)$ may be regarded as a $q$-analog of the Capelli polynomial for the algebra $\mathrm{U}_{q}^{\mathrm{tw}}\left(\mathfrak{o}_{N}\right)$. It would be interesting to find its eigenvalues in the irreducible representations and to get the corresponding $q$-analogs of the Capelli identities; cf. 27, [13, 15, 34. 
Example 4.11. If $N=3$ then

$$
C(u)=(u+q)\left[(u+q)\left(u+q^{3}\right)-q^{2} u C\right]
$$

where

$$
C=s_{21}^{2}+q^{2} s_{32}^{2}+s_{31}^{2}-q s_{21} s_{32} s_{31} .
$$

The following corollary provides a characteristic identity for the algebra $\mathrm{U}_{q}^{\mathrm{tw}}\left(\mathfrak{o}_{N}\right)$; cf. [27, [30].

Corollary 4.12. We have the identity

$$
C\left(-q^{2 N-3} \bar{S} S^{-1}\right)=0
$$

Proof. Introduce the quantum comatrix $\widehat{S}(u)$ by the formula

$$
\widehat{S}(u) S\left(u q^{-2 N+2}\right)=\operatorname{sdet} S(u)
$$

Explicit expressions for the matrix elements of the matrix $\widehat{S}(u)$ can be found from the recurrence relation for the elements $s_{a_{1} \cdots a_{r-1}, b_{r}}^{a_{1} \cdots a_{r}}(u)$; see the end of the proof of Theorem 4.7. Using these expressions and applying the evaluation homomorphism to both sides of (4.49) we get

$$
C(u)=\widehat{C}(u)\left(\bar{S}+u q^{-2 N+3} S\right),
$$

where $\widehat{C}(u)$ is a polynomial in $u$ with coefficients in the algebra $\mathrm{U}_{q}^{\text {tw }}\left(\mathfrak{o}_{N}\right) \otimes$ End $\mathbb{C}^{N}$. This completes the proof.

\section{References}

[1] D. Arnaudon, J. Avan, N. Crampé, L. Frappat, E. Ragoucy, R-matrix presentation for super-Yangians $Y(\operatorname{osp}(m \mid 2 n))$, J. Math. Phys. 44 (2003), 302-308.

[2] V. Chari and A. Pressley, A guide to quantum groups, Cambridge University Press, 1994.

[3] I. V. Cherednik, A new interpretation of Gelfand-Tzetlin bases, Duke Math. J. 54 (1987), 563-577.

[4] G. W. Delius, N. J. MacKay, B. J. Short, Boundary remnant of Yangian symmetry and the structure of rational reflection matrices, Phys. Lett. B 522 (2001), 335-344; Erratum ibid. B 524 (2002) 401. 
[5] G. W. Delius and N. J. MacKay, Quantum group symmetry in sine-Gordon and affine Toda field theories on the half-line, Comm. Math. Phys. 233 (2003), 173190.

[6] M. S. Dijkhuizen and M. Noumi, A family of quantum projective spaces and related q-hypergeometric orthogonal polynomials, Trans. AMS 350 (1998), 32693296.

[7] M. S. Dijkhuizen, M. Noumi and T. Sugitani, Multivariable Askey-Wilson polynomials and quantum complex Grassmannians, Fields Int. Commun. 14 (1997), $167-177$.

[8] J. Ding, Spinor representations of $U_{q}(\hat{\mathfrak{g} l}(n))$ and quantum boson-fermion correspondence, Comm. Math. Phys. 200 (1999), 399-420.

[9] V. G. Drinfeld, Hopf algebras and the quantum Yang-Baxter equation, Soviet Math. Dokl. 32 (1985), 254-258.

[10] V. G. Drinfeld, A new realization of Yangians and quantized affine algebras, Soviet Math. Dokl. 36 (1988), 212-216.

[11] E. Frenkel and E. Mukhin, The Hopf algebra $\operatorname{Rep} U_{q} \widehat{\mathfrak{g l}}_{\infty}$, Selecta Math. (N.S.) 8 (2002), 537-635.

[12] A. M. Gavrilik and A. U. Klimyk, q-deformed orthogonal and pseudo-orthogonal algebras and their representations, Lett. Math. Phys. 21 (1991), 215-220.

[13] A. M. Gavrilik and N. Z. Iorgov, On Casimir elements of q-algebras $U_{q}^{\prime}\left(\mathrm{so}_{n}\right)$ and their eigenvalues in representations, in 'Symmetry in nonlinear mathematical physics', Proc. Inst. Mat. Ukr. Nat. Acad. Sci. 30, Kyiv, 1999, pp. 310-314.

[14] A. M. Gavrilik, N. Z. Iorgov and A. U. Klimyk, Nonstandard deformation $U_{q}^{\prime}\left(\mathrm{so}_{n}\right)$ : the embedding $U_{q}^{\prime}\left(\mathrm{so}_{n}\right) \subset U_{q}\left(\mathrm{sl}_{\mathrm{n}}\right)$ and representations, in 'Symmetries in science', X (Bregenz, 1997). Plenum, New-York, 1998, pp. 121-133.

[15] M. Havlíček, A. U. Klimyk and S. Pošta, Central elements of the algebras $U_{q}^{\prime}\left(\mathrm{so}_{m}\right)$ and $U_{q}\left(\right.$ iso $\left._{m}\right)$, Czechoslovak J. Phys. 50 (2000), 79-84.

[16] J. E. Humphreys, Introduction to Lie algebras and Representation Theory, Springer, New York, 1972.

[17] A. G. Izergin and V. E. Korepin, A lattice model related to the nonlinear Schrödinger equation, Sov. Phys. Dokl. 26 (1981) 653-654. 
[18] M. Jimbo, A q-difference analogue of $\mathrm{U}(\mathfrak{g})$ and the Yang-Baxter equation, Lett. Math. Phys. 10 (1985), 63-69.

[19] M. Jimbo, A q-analogue of $U_{q}(\mathfrak{g l}(N+1))$, Hecke algebra and the Yang-Baxter equation, Lett. Math. Phys. 11 (1986), 247-252.

[20] P. P. Kulish and E. K. Sklyanin, Quantum spectral transform method: recent developments, in 'Integrable Quantum Field Theories', Lecture Notes in Phys. 151 Springer, Berlin-Heidelberg, 1982, pp. 61-119.

[21] G. Letzter, Symmetric pairs for quantized enveloping algebras, J. Algebra 220 (1999), 729-767.

[22] G. Letzter, Coideal subalgebras and quantum symmetric pairs, in 'New directions in Hopf algebras', Math. Sci. Res. Inst. Publ. 43, Cambridge Univ. Press, Cambridge, 2002, pp. 117-165.

[23] G. Letzter, Quantum symmetric pairs and their zonal spherical functions, preprint math.QA/0204103.

[24] A. Liguori, M. Mintchev and L. Zhao, Boundary exchange algebras and scattering on the half line, Comm. Math. Phys. 194 (1998), 569-589.

[25] M. Mintchev, E. Ragoucy and P. Sorba, Spontaneous symmetry breaking in the $g l(N)-N L S$ hierarchy on the half line, J. Phys. A 34 (2001), 8345-8364.

[26] A. I. Molev, Stirling partitions of the symmetric group and Laplace operators for the orthogonal Lie algebra, Discrete Math. 180 (1998), 281-300.

[27] A. I. Molev, Yangians and their applications, in "Handbook of Algebra", Vol. 3, (M. Hazewinkel, Ed.), Elsevier, 2003.

[28] A. Molev, M. Nazarov and G. Olshanski, Yangians and classical Lie algebras, Russian Math. Surveys 51:2 (1996), 205-282.

[29] A. I. Molev and E. Ragoucy, Representations of reflection algebras, Rev. Math. Phys. 14 (2002), 317-342.

[30] M. Nazarov and V. Tarasov, Yangians and Gelfand-Zetlin bases, Publ. RIMS, Kyoto Univ. 30 (1994), 459-478.

[31] M. Noumi, Macdonald's symmetric polynomials as zonal spherical functions on quantum homogeneous spaces, Adv. Math. 123 (1996), 16-77. 
[32] M. Noumi and T. Sugitani, Quantum symmetric spaces and related q-orthogonal polynomials, in 'Group Theoretical Methods in Physics (ICGTMP)' (Toyonaka, Japan, 1994), World Sci. Publishing, River Edge, N.J. (1995), pp. 28-40.

[33] M. Noumi, T. Umeda and M. Wakayama, A quantum dual pair $\left(\mathfrak{s l}_{2}, \mathfrak{o}_{n}\right)$ and the associated Capelli identity, Lett. Math. Phys. 34 (1995), 1-8.

[34] M. Noumi, T. Umeda and M. Wakayama, Dual pairs, spherical harmonics and a Capelli identity in quantum group theory, Compos. Math. 104 (1996), 227-277.

[35] G. Olshanski, Twisted Yangians and infinite-dimensional classical Lie algebras, in 'Quantum Groups' (P. P. Kulish, Ed.), Lecture Notes in Math. 1510, Springer, Berlin-Heidelberg, 1992, pp. 103-120.

[36] N. Yu. Reshetikhin, L. A. Takhtajan and L. D. Faddeev, Quantization of Lie Groups and Lie algebras, Leningrad Math. J. 1 (1990), 193-225.

[37] E. K. Sklyanin, Boundary conditions for integrable quantum systems, J. Phys. A21 (1988), 2375-2389. 